\title{
Topological and chemical characteristics of turbulent flames at MILD conditions
}

\author{
Dimitris M. Manias ${ }^{\mathrm{a}, \mathrm{b}}$, Efstathios-Al. Tingas ${ }^{\mathrm{a}, \mathrm{d}, *}$, Yuki Minamoto ${ }^{\mathrm{c}}$, Hong G. Im $^{\mathrm{a}}$ \\ ${ }^{a}$ Clean Combustion Research Center (CCRC), King Abdullah University of Science and Technology \\ (KAUST), Thuwal, 23955-6900, Saudi Arabia. \\ ${ }^{b}$ Department of Mechanics, School of Applied Mathematics and Physical Sciences, National Technical \\ University of Athens, 15773 Athens, Greece \\ ${ }^{c}$ Department of Mechanical Engineering, Tokyo Institute of Technology, Meguro, Tokyo 152-8550, Japan \\ ${ }^{d}$ Perth College, University of the Highlands and Islands (UHI), Crieff Rd, Perth PH1 2NX, UK
}

\begin{abstract}
Dominant physical processes that characterize the combustion of a lean methane/air mixture, diluted with exhaust gas recirculation (EGR), under turbulent MILD premixed conditions are identified using the combined approach of Computational Singular Perturbation (CSP) and Tangential Stretching Rate (TSR). TSR is a measure to combine the time scale and amplitude of all active modes and serves as a rational metric for the true dynamical characteristics of the system, especially in turbulent reacting flows in which reaction and turbulent transport processes compete. Applied to the MILD conditions where the flame structures exhibit nearly distributed combustion modes, the TSR metric was found to be an excellent diagnostic tool to depict the regions of important activities. In particular, the analysis of turbulent DNS data revealed that the system's dynamics is mostly dissipative in nature, as the chemically explosive modes are largely suppressed by the dissipative action of transport. On the other hand, the convective transport associated with turbulent eddies play a key role in bringing the explosive nature into the system. In the turbulent MILD conditions under study, the flame structure appears nearly in the distributed combustion regime, such that the conventional statistics conditioned over the progress variable becomes inappropriate, but TSR serves as an automated and systematic way to depict the topology of such complex flames. In addition, further analysis of the CSP modes revealed a strong competition between explosive and dissipative modes, the former favored by hydrogen-related reactions and the convection of $\mathrm{CH}_{4}$, and the latter by carbon-related processes. This competition results in a much smaller region of explosive dynamics in contrast to the widespread existence of explosive modes.
\end{abstract}

Keywords: MILD combustion, Turbulent flames, CSP, TSR

\section{Introduction}

Developing modern combustion engines needs to address stringent environmental regulations such as efficiency, pollutants and noise. As a promising approach to achieve high

${ }^{*}$ Corresponding author: stathis.tingas@kaust.edu.sa 
efficiency and reduced emissions, moderate or intense low-oxygen dilution (MILD) combustion [1] has been investigated. Also referred to as flameless combustion because of the absence of a well determined visible flame, in MILD conditions, a reactant mixture is preheated and highly diluted by using exhaust gas or flue gas to achieve stable combustion and relatively low flame temperature. This leads to lower thermal NOx formation and noise, as well as high combustion efficiency [2].

MILD combustion conditions have been realized in the laboratory by air preheating with exhaust gas recirculation [3]. Chemical characterization has been conducted such as ignition delays and the oxidation pathways for methane oxidation at MILD conditions [4, 5]. The effect of $\mathrm{H}_{2} \mathrm{O}$ and $\mathrm{CO}_{2}$ addition to methane mixtures has also been studied numerically [6-8] by considering canonical reactors for autoignition problems.

Considering the preheated reactant mixture being more reactive, the characteristics of transport-chemistry interaction in turbulent flames at MILD conditions are distinct from those at normal conditions. Experimental measurements suggest a uniform and relatively distributed combustion under MILD conditions, while the $\mathrm{OH}$ images present a contrasting view of well-defined thin reaction zones, making it difficult to categorize in the standard "turbulent combustion regime" context [9-14]. Three-dimensional direct numerical simulation (3D DNS) of turbulent methane flame at MILD conditions has been conducted [15], reporting regions with strong chemical activity along with the interactions between different reaction zones at different dilution levels [16]. Extended studies on the structure of the MILD reaction zones and their relation to conventional turbulent flames have also been carried out [17, 18], along with their modeling implications[19]. More recently, MILD reaction zone structures in the physical and mixture fraction spaces were investigated by using the flame index approach [20]. Non-EGR-type MILD combustion was also simulated by means of 3D DNS in a mixing layer configuration to study the effect of preferential diffusion and heat losses on local ignition delay in a shear-driven turbulence [21].

These previous studies addressed key issues for MILD combustion, such as dominant reaction pathways depending on different diluent gases at varying dilution levels, and the morphology of MILD reaction zones exhibiting multi-mode combustion [16]. From a theoretical standpoint, this implies changes in the characteristic physical time scales driving the system dynamics. For example, the preheat zone of the flame is usually characterized by the balance between convection and diffusion. In MILD condition, due to the increased chemical reactivity, the balance may be shifted to an increased role of reactions, although the dominant chemical pathways in this region may be different from those in the main reaction zone. As such, proper characterization of the structure of turbulent flames at MILD conditions may require a significantly different approach depending on the thermochemical conditions of the reactant mixture.

To this end, the mathematical tools provided by the computational singular perturbation (CSP) [22] method, which is an algorithmic method of asymptotic analysis, serves as a systematic tool to analyze the physics involving multiple chemical and transport processes with different time scales, by identifying the important dynamical characteristics driven by the key processes. One of the major advantages of CSP is that it can identify locally the dominant processes that contribute to the dominant modes driving the slow dynamics of the system and their related timescales. This allows an objective characterization of the competing processes without resorting to empirical intuition, thus leading to clear physical 
understanding. These timescales are systematically obtained by applying the CSP algorithm to the governing equations of the system, without any a priori assumptions or approximations about dominant physical phenomenon in the localized region (e.g. flame propagation or autoignition). Furthermore, the tangential stretching rate (TSR) [23], a unified and extended metric to represent the significance of the dominant modes, can be employed as a diagnostic tool to identify various dynamical characteristics of a reactive-transport system. TSR adopts major concepts from the CSP framework and uses its algorithmic tools in order to identify the explosive/dissipative states of the system, by accounting for the effect of all CSP modes.

In the present study, the combined approach of CSP and TSR and their algorithmic tools are used to analyze the dynamics of an EGR-type methane/air premixed flame under MILD combustion conditions, that was previously simulated in the context of 3D DNS with a complex chemistry $[16,24]$. The objective of this work is twofold: (i) to investigate the topology of the system, and (ii) to investigate the effect of turbulence on the system's dynamics and the dominant chemical pathways. For the purpose of the second goal, a 1D laminar case is also considered and analyzed extensively.

The structure of the paper is as follows. First, a description of the theoretical framework and the algorithmic tools is provided. As a baseline study, both a 0D ignition and 1D laminar cases are then analyzed with the CSP tools to provide a generic chemical and transport structure. In Section 4, the analysis of the turbulent case starts with a brief description of the computational setup and the extensive CSP analysis follows in Section 5. Finally, in Section 6 the dominant chemical pathways that are responsible for the competitive action between explosive and dissipative modes and the effect of turbulence are described in detail.

\section{Theoretical framework of analysis}

A general form of the species and temperature governing equations that describe the evolution of a reacting system without transport is written as:

$$
\frac{d \boldsymbol{z}}{d t}=\boldsymbol{g}(\boldsymbol{z})=\sum_{k=1}^{2 K} \hat{\mathbf{S}}_{k} R^{k}=\hat{\mathbf{S}}_{1} R^{1}+\hat{\mathbf{S}}_{2} R^{2}+\ldots+\hat{\mathbf{S}}_{2 K} R^{2 K}
$$

where $\boldsymbol{z}$ is a column vector with $(N+1)$ solution variables including $N$ species mass fractions $(\boldsymbol{y})$ and temperature $(T), \boldsymbol{g}(\boldsymbol{z})$ the chemical reaction source term, and $\hat{\mathbf{S}}_{k}$ and $R^{k}$ are the $(N+$ 1)-dimensional generalized stoichiometric column vector and the reaction rate, respectively, of the $k$-th unidirectional reaction of a total of $K$ reversible reactions. According to the CSP approach [22, 25], Eq. (1) is transformed into:

$$
\frac{d \boldsymbol{z}}{d t}=\boldsymbol{g}(\boldsymbol{z})=\boldsymbol{a}_{1} f^{1}+\boldsymbol{a}_{2} f^{2}+\ldots+\boldsymbol{a}_{N} f^{N}+\boldsymbol{a}_{N+1} f^{N+1}
$$

where $\boldsymbol{a}_{i}$ is the $(N+1)$-dimensional CSP basis column vector and $f^{i}=\boldsymbol{b}^{i} \cdot \boldsymbol{g}(\boldsymbol{z})$ is the related amplitude, where $\boldsymbol{b}^{i}$ denotes the dual basis row vector $\left(\boldsymbol{b}^{i} \cdot \boldsymbol{a}_{j}=\delta_{j}^{i}\right)[22,25]$. At leading order, $\boldsymbol{a}_{i}$ and $\boldsymbol{b}^{i}(i=1, N+1)$ are approximated, respectively, by the right and left eigenvectors of the Jacobian $\boldsymbol{J}$ of $\boldsymbol{g}(\boldsymbol{z})$ [22, 25-28]. The timescale of each CSP mode is defined as the inverse norm of the corresponding eigenvalue, i.e., $\tau_{i}=\left|\lambda_{i}\right|^{-1}$, where $\lambda_{i}=\boldsymbol{\beta}^{i} \cdot \boldsymbol{J} \cdot \boldsymbol{\alpha}_{i}$, and $\boldsymbol{\alpha}_{i}$ and $\boldsymbol{\beta}^{i}$ are the $i$-th right (column) and left (row) eigenvectors of $\boldsymbol{J}$, respectively [29]. A mode is 
determined to be explosive if its eigenvalue is positive, or dissipative otherwise. An explosive mode drives the dynamical system away from equilibrium, while a dissipative mode drives the system towards equilibrium [30].

Based on a proper threshold between fast and slow time scales [31], the first $M$ timescales that are of dissipative nature and faster are referred to as exhausted, and Eq. 2 is reduced to $[22,25]$ :

$$
f^{m} \approx 0 \quad(m=1, \ldots M) \quad \frac{d \mathbf{z}}{d t} \approx \sum_{i=M+1}^{N+1} \mathbf{a}_{i} f^{i},
$$

where the $M$ algebraic expressions define a slow invariant manifold (SIM), on which the solution evolves according to the reduced set of the ODEs [32], which is free of the fast timescales and its dynamics is mainly characterized by the fastest of the slow timescales (mode $M+1$ ). In the present study, the selection of $M$ is determined by the revised criterion provided in Ref. [33].

Although the transformation of the initial system to the CSP-basis space is purely mathematical, each CSP mode is associated with a definite relation between a number of relevant physical and chemical processes, identified through various algorithmic tools. For example, considering that the eigenvalue of the $n$-th CSP mode $(1 \leq n \leq N+1)$ is expressed as a sum of a total of $2 K$ components, each referring to a reaction (process) as:

$$
\lambda_{n}=\boldsymbol{\beta}^{n} \sum_{k=1}^{2 K} \nabla\left(\hat{\mathbf{S}}_{k} R^{k}\right) \boldsymbol{\alpha}_{n}=c_{1}^{n}+\ldots+c_{2 K}^{n},
$$

the timescale participation index (TPI) is defined as a measure of the relative contribution of each process to the timescale of the $n$-th mode, $\tau_{n}[34-37]$ :

$$
T P I_{k}^{n}=\frac{c_{k}^{n}}{\left|c_{1}^{n}\right|+\ldots+\left|c_{2 K}^{n}\right|}
$$

where $n=1, \ldots, N+1$ and $k=1, \ldots, 2 K$. According to the above definition the sign of TPI is consistent with the corresponding mode; a positive (negative) TPI implies that the $k$-th reaction contributes to an explosive (dissipative) character of $\tau_{n}$ [38].

The CSP approach was later extended to a system of partial differential equations (PDEs) to include transport terms [39, 40]. For a general reactive-transport system, Eq. (1) is revised into the form:

$$
\frac{\partial \boldsymbol{z}}{\partial t}=\boldsymbol{L}(\boldsymbol{z})+\boldsymbol{g}(\boldsymbol{z})
$$

where $\boldsymbol{L}(\boldsymbol{z})$ is the a spatial operator representing convection and/or diffusion. By introducing the CSP basis vectors, Eq. 6 is again cast in the form [39-43]:

$$
\frac{\partial \boldsymbol{z}}{\partial t}=\sum_{i=1}^{N+1} \mathbf{a}(\boldsymbol{z}) h^{i}(\boldsymbol{z})
$$

where the amplitude $h^{n}$ of the $n$-th mode is defined as:

$$
h^{n}=\boldsymbol{b}^{n} \cdot(\boldsymbol{L}(\boldsymbol{z})+\boldsymbol{g}(\boldsymbol{z})) .
$$


and is expressed as a sum of $2 K+(N+1)$ terms as:

$$
h^{n}=\boldsymbol{b}^{n} \cdot(\boldsymbol{L}(\boldsymbol{z})+\boldsymbol{g}(\boldsymbol{z}))=\sum_{j=1}^{N+1} b_{j}^{n} L_{j}+\sum_{k=1}^{2 K} \boldsymbol{b}^{n} \cdot \hat{\mathbf{S}}_{k} R^{k}=\sum_{l=1}^{2 K+N+1} \beta_{l}^{n} .
$$

Therefore, the relative contribution of each process (either reaction or transport) to the amplitude of each mode is quantified using the amplitude participation index (API) [44]:

$$
A P I_{k}^{n}=\frac{\beta_{k}^{n}}{\sum_{j=1}^{2 K+N+1}\left|\beta_{j}^{n}\right|} .
$$

where $n=1, \ldots, N+1$ and $k=1, \ldots, 2 K+N+1$. Note that the amplitudes of all modes are set to be always positive by properly adjusting the signs of the related right and left eigenvectors [45]. As such, a positive (negative) sign of API indicate the increasing (decreasing) effect of each process on the amplitude of each mode.

The success of the CSP approach has been tested in a wide range of combustion applications, from model simplification $[28,46]$ and reduction $[47,48]$ to the analysis of highly complex kinetics models in auto-igniting systems $[49,50]$, premixed, non-premixed and partially premixed flames [28, 51, 52], PSR [53], with datasets obtained from RANS [27], LES [54] and DNS [55-57], along with the development of algorithmic methods for the characterization of the flame topology $[56,57]$ and ignition $[58,59]$ or pollutant [60] control. By properly identifying the characteristic mode $(M+1)$ that drives the slow dynamics of the system, one can determine the dominant physical processes and components contributing to the characteristic mode. However, a standard CSP analysis to identify the characteristic mode based on its time scale may sometimes lead to a misleading physical interpretation, since the amplitude of mode $M+1$ may be small, as demonstrated in our recent studies $[57,61,62]$. To address this issue, the tangential stretching rate (TSR) [23, 63] has been introduced as a weighted average of the eigenvalues with the corresponding amplitudes. For a system without transport, the reactive TSR is defined as:

$$
\omega_{\tilde{\tau}_{R}}=\sum_{i=1}^{N+1} W_{i, R} \lambda_{i}
$$

where the weights $W_{i, R}$ are determined by:

$$
W_{i, R}=\frac{f^{i}}{\mathrm{~g}} \sum_{k=1}^{N+1} \frac{f^{k}}{\mathrm{~g}}\left(\boldsymbol{a}_{k} \cdot \boldsymbol{a}_{i}\right) .
$$

and $\mathrm{g}$ is the norm of $\boldsymbol{g}$. For a reactive-transport system, the extended TSR is defined as [63]:

$$
\omega_{\tilde{\tau}_{R+T}}=\sum_{i=1}^{N+1} W_{i, R+T} \lambda_{i}
$$

where

$$
W_{i, R+T}=\frac{h^{i}}{\mathrm{~L}+\mathrm{g}} \sum_{k=1}^{N+1} \frac{h^{k}}{\mathrm{~L}+\mathrm{g}}\left(\boldsymbol{a}_{k} \cdot \boldsymbol{a}_{i}\right)
$$


and $\mathrm{L}$ is the norm of $\boldsymbol{L}$.

As such, TSR serves as a true indicator to describe whether the system is driven away from SIM (explosive) or approaching to SIM (dissipative), as the resultant effect of all relevant modes. In general, a number of modes contribute to the TSR dynamics. The contribution of each mode to TSR is determined by the TSR participation index (TSR-PI)[63], defined for a reactive system without transport as:

$$
V_{n}^{R}=\operatorname{sign}\left(\operatorname{Re}\left(\lambda_{n}\right)\right) \frac{W_{n, R}\left|\lambda_{n}\right|}{\sum_{i=1}^{N+1}\left|W_{i, R}\right| \lambda_{i}||} .
$$

and for a reactive-transport system as:

$$
V_{n}^{R+T}=\operatorname{sign}\left(\operatorname{Re}\left(\lambda_{n}\right)\right) \frac{W_{n, R+T}\left|\lambda_{n}\right|}{\sum_{i=1}^{N+1}\left|W_{i, R+T}\right| \lambda_{i}||}
$$

According to the sign convention, the largest value of TSR-PI quantifies the contribution of the $n$-th mode to the dynamic growth (positive TSR-PI) or decay (negative TSR-PI) of the system.

TSR-PI reveals the contribution by different CSP modes. To identify the contribution of different processes (reaction or transport) to the TSR, the TSR-API is defined as the product of TSR-PI (Eqs. 15, 16) and API (Eq. 10):

$$
H_{k}^{R+T}=V_{n}^{R+T} \times\left|A P I_{k}^{n}\right|
$$

for reactive-transport systems. Eq. 17 provides an effective local assessment of the contribution of chemical kinetics versus transport to the slow dynamics of the system. Thus, the sum of the contributions of all the reactions $\left(\sum_{k=1}^{2 K} H_{k}^{R+T}\right.$ for all kinetic terms) quantifies the net role of kinetics $\left(\mathrm{H}_{C h e m}^{R+T}\right)$, the sum of the contributions of all the diffusive/convective terms ( $\sum_{k=2 K+1}^{2 K+N+1} H_{k}^{R+T}$ for all diffusive/convective terms) quantifies the net role of diffusion $\left(\mathrm{H}_{\text {Diff }}^{R+T}\right) /$ convection $\left(\mathrm{H}_{\text {Conv }}^{R+T}\right)$, respectively, and the sum of the contributions of all the transport terms $\left(\sum_{k=2 K+1}^{2 K+N+1} H_{k}^{R+T}\right.$ for all transport terms) quantifies the role of transport $\left(\mathrm{H}_{\text {Tran }}^{R+T}\right)$ $[56,57]$.

Due to its large dynamic range, in the following presentation both TSR and the eigenvalues are represented by their logarithms, i.e.:

$$
\begin{gathered}
\Omega_{R}=\operatorname{Sign}\left(\omega_{\tilde{\tau}_{R}}\right) \cdot \log _{10}\left|\omega_{\tilde{\tau}_{R}}\right| \\
\Omega_{R+T}=\operatorname{Sign}\left(\omega_{\tilde{\tau}_{R+T}}\right) \cdot \log _{10}\left|\omega_{\tilde{\tau}_{R+T}}\right| \\
\Lambda_{i}=\operatorname{Sign}\left(\lambda_{i}\right) \cdot \log _{10}\left|\lambda_{i}\right|
\end{gathered}
$$

Note that $\Omega_{R+T}=\Omega_{R}$ implies that the role of transport is insignificant and the chemical source term is dominant in the generation of $\Omega_{R+T}$. On the other hand, when $\Omega_{R+T} \neq \Omega_{R}$, no definite statement can be made about the role of transport, and the $\mathrm{H}_{\text {Chem }}^{R+T}$ and $\mathrm{H}_{\text {Tran }}^{R+T}$ indices should be used instead. This is because the inclusion of transport in the amplitudes of the modes alters (increases/decreases) the weights of all modes (explosive/dissipative). For instance, in a region where $\Omega_{R+T}>0$ and $\Omega_{R}<0$, a positive value of $\Omega_{R+T}$ may occur either 
by an increase in the amplitude of an explosive mode due to transport, or by a decrease in the largest amplitude of the dissipative mode, or a combination of both. In fact, in the last case, $\mathrm{H}_{\text {Chem }}^{R+T}$ may be large and $\mathrm{H}_{\text {Tran }}^{R+T}$ small, despite that $\Omega_{R+T}>0$ and $\Omega_{R}<0$.

For all presented results related to the CSP analysis, the CSPTk package [64] integrated with the TChem package [65] for thermo-kinetic database management was employed.

\section{Autoignition and laminar flame dynamics}

As a baseline case study, the CSP-TSR analysis was performed in a homogeneous and adiabatic autoignition and a one-dimensional freely propagating laminar flame problems. For all simulations, the skeletal chemical kinetics mechanism by Smooke with 16 species and 35 elementary reactions [66] was used, and the results were further confirmed with the GRI 3.0 detailed mechanism [67]. The 0D and 1D simulations were performed using the ChemkinPro software [68]. The initial/inflow thermochemical conditions same as the turbulent DNS case presented later were used: initial pressure and temperature; $T(0)=1500 \mathrm{~K}, p(0)=1 \mathrm{~atm}$, $Y_{\mathrm{CH}_{4}}=3.471 \mathrm{E}-03, Y_{\mathrm{O}_{2}}=2.8718 \mathrm{E}-02, Y_{\mathrm{H}_{2} \mathrm{O}}=9.3246 \mathrm{E}-02, Y_{\mathrm{CO}_{2}}=1.0813 \mathrm{E}-01, Y_{\mathrm{N}_{2}}=7.6643 \mathrm{E}-01$, where $Y_{i}$ the mass fraction of the $i$-th species. In these conditions the ignition delay time, based on the maximum temperature rise rate, as measured from a constant pressure batch reactor is $t_{i g n}=9.74 \mathrm{~ms}$, and the laminar flame speed of a freely propagating unstrained flame is $S_{L}=1.67 \mathrm{~m} / \mathrm{s}$. The flame structure analysis was presented in terms of a temperaturebased progress variable, $c_{T}$, defined as $c_{T}=\left(T-T_{r}\right) /\left(T_{p}-T_{r}\right)$, where $T_{r}$ and $T_{p}$ are the temperature values of the reactants and products, respectively. For future reference, most significant reactions to the dynamics of the system are summarized in Table 1 . Note that when the reactant temperature is larger than its autoignition temperature, the value of the flame speed may depend on the computational domain size. In this study, the laminar flame was computed using a domain size of $10 \mathrm{~mm}$, which is the length employed for the DNS computations, hence allowing a meaningful comparison against the turbulent case [18].

Table 1: The most significant reactions to the dynamics of the system in all examined cases.

\begin{tabular}{rlll}
\hline 1. & $\mathrm{H}+\mathrm{O}_{2} \rightarrow \mathrm{OH}+\mathrm{O}$ & 20. & $\mathrm{CH}_{3}+\mathrm{H}_{2} \mathrm{O} \rightarrow \mathrm{CH}_{4}+\mathrm{OH}$ \\
2. & $\mathrm{OH}+\mathrm{O} \rightarrow \mathrm{H}+\mathrm{O}_{2}$ & 22. & $\mathrm{CH}_{2} \mathrm{O}+\mathrm{H} \rightarrow \mathrm{HCO}+\mathrm{H}_{2}$ \\
16. & $\mathrm{CH}_{3}+\mathrm{H}(+\mathrm{M}) \rightarrow \mathrm{CH}_{4}(+\mathrm{M})$ & 23. & $\mathrm{CH}_{2} \mathrm{O}+\mathrm{OH} \rightarrow \mathrm{HCO}+\mathrm{H}_{2} \mathrm{O}$ \\
17. & $\mathrm{CH}_{4}+\mathrm{H} \rightarrow \mathrm{CH}_{3}+\mathrm{H}_{2}$ & 25. & $\mathrm{HCO}+\mathrm{M} \rightarrow \mathrm{CO}+\mathrm{H}+\mathrm{M}$ \\
19. & $\mathrm{CH}_{4}+\mathrm{OH} \rightarrow \mathrm{CH}_{3}+\mathrm{H}_{2} \mathrm{O}$ & 26. & $\mathrm{CH}_{3}+\mathrm{O}_{2} \rightarrow \mathrm{CH}_{3} \mathrm{O}+\mathrm{O}$ \\
\hline
\end{tabular}

Figure 1(a) shows the evolution of the species mass fractions for the 0D and 1D cases, both of which are plotted in terms of $c_{T}$. The behavior among different species for $0 \mathrm{D}$ and 1D results are very similar, with $0 \mathrm{D}$ case showing a slightly faster fuel consumption and radical production in the absence of diffusive processes that tend to dissipate the produced radicals. The comparison of the eigenvalues $\Lambda_{i}$ between the 0D and 1D cases shown in Fig. 1(b) also indicates that the key chemical pathways and their time scales are nearly identical between $0 \mathrm{D}$ and $1 \mathrm{D}$ cases, as would be expected from the two cases with identical initial/upstream thermodynamic conditions. In terms of TSR, however, i.e. when the amplitudes of the characteristic chemical modes are taken into consideration, an important difference reveals 
that the ranges of positive $\Omega_{R}$ are significantly diminished, implying that the chemically explosive modes are attenuated by the dissipative transport in the upstream preheat zone of the 1D flame, leaving a much narrower explosive region where heat release rate is maximum. This is consistent with the behavior of hydrogen-air flames reported in Ref. [57]. It is evident that the TSR provides a better dynamical characteristics in the flames when transport effects are present.

To further account for the role of transport, the extended TSR, $\Omega_{R+T}$, is investigated. It is first noted that, for a steady problem, the right hand side of Eq. 8 vanishes, leading to a trivial result of $h^{n}=0$. Therefore, a more proper way to characterize the explosive dynamics of the steady flame is to split the convective and diffusive operators and only consider the amplitudes associated with reaction and diffusion. This is equivalent to examining the Lagrangian dynamics $(D \boldsymbol{z} / D t)$, which makes sense for a steady premixed flame with a unique mean convective velocity. As will be discussed later in the turbulent flame analysis, the convective operator for turbulent flow conditions will be dominated by the turbulent component, and thus Eq. 8 serves as a proper metric for the amplitudes.

Figure 2 shows the behavior of the reactive TSR $\left(\Omega_{R}\right)$ and extended TSR $\left(\Omega_{R+D}\right)$ in the physical (Fig. 2a) and the progress variable $\left(c_{T}\right)$ (Fig. 2b) spaces, overlaid with the heat release rate $(\mathrm{HRR})$ profile. The temperature evolution is also overlaid in Fig. 2a. From early in the process, $\Omega_{R+D}$ becomes positive, (in contrast to $\Omega_{R}$ which remains negative), highlighting the importance of transport to the explosive dynamics at the preheat zone. The $\Omega_{R+D}$ profile nearly follows the evolution of $\Omega_{R}$ in the 0D ignition problem (See Fig. 1(b)), which indicates that, while the reaction alone cannot, the net effect of combined diffusivereactive leads to the explosive dynamics in the preheat zone of the $1 \mathrm{D}$ flame. Fig. 2(b) further shows the TSR-API, indicating that the contributions from reaction $\left(H_{\text {Chem }}^{R+T}\right)$ and diffusion $\left(H_{D i f f}^{R+T}\right)$ are comparable in the preheat zone where $c_{T}<0.3$, while the diffusive contribution is higher in the upstream preheat zone. This suggests that the information obtained from both $\Omega_{R}$ and $\Omega_{R+T}$ are valuable in identifying the reactive and preheat zones of the laminar flames, and the analysis will be applied to turbulent flames.

The above results suggest that in both the $0 \mathrm{D}$ and $1 \mathrm{D}$ cases, the fast explosive mode is the characteristic one in the flame front region. As such, Fig. 3 further shows the important processes contributing to the explosive dynamics in the preheat zone of the flame, in terms of (a) TPI and (b) API. TPI shows a nearly similar behavior between 0D and 1D in terms of the dominant reaction steps and their magnitudes. The dominant chemical pathways are found to be as expected: the chain branching reaction $\mathrm{R} 1\left(\mathrm{H}+\mathrm{O}_{2} \rightarrow \mathrm{OH}+\mathrm{O}\right)$ is the largest contributor favoring the explosive dynamics, while the major opposition is produced by reactions $\mathrm{R} 16\left(\mathrm{CH}_{3}+\mathrm{H}(+\mathrm{M}) \rightarrow \mathrm{CH}_{4}(+\mathrm{M})\right)$ and $\mathrm{R} 2$ (the reverse of $\left.\mathrm{R} 1\right)$. Note also that reaction $\mathrm{R} 26\left(\mathrm{CH}_{3}+\mathrm{O}_{2} \rightarrow \mathrm{CH}_{3} \mathrm{O}+\mathrm{O}\right)$ is the main contributor to the explosive character at very low $c_{T}$ values $\left(c_{T}<0.01\right)$, which along with $\mathrm{R} 16$ indicate the importance of $\mathrm{CH}_{3}$ chemistry in that early stage of ignition/flame. As for the API, the 0D case results are not shown here, as the behavior is nearly identical to those with TPI. In contrast, the API for the $1 \mathrm{D}$ flame clearly shows the contributions from diffusion, especially that of $\mathrm{H}, \mathrm{H}_{2}$ and $\mathrm{CH}_{3}$, in addition to reaction $\mathrm{R} 1$. The effect of $\mathrm{H}_{2}$ and $\mathrm{CH}_{3}$, which are fast diffusing species from the original fuels, dominates in the far upstream region, while the diffusion of $\mathrm{H}$ atom is more widespread throughout the preheat zone.

Before the analysis of the turbulent case, we highlight the differences in the structure 
of the explosive dynamics between the present MILD flame versus a non-diluted laminar flame at $\phi=0.8$ and upstream temperature at $300 \mathrm{~K}$. Figure 4 shows the comparison of both $\Lambda_{e}$ and $\Omega_{R+D}$ between the MILD condition flame shown earlier and the normal laminar flame case. First, it is evident that the explosive dynamics is significantly suppressed in the normal flame due to the lower temperature in the preheat zone, and large values of $\Lambda_{e}$ are more confined only in the heat release region. In contrast, for the MILD combustion the overall explosive dynamics are shifted further upstream and widely present throughout the upstream region.

In summary, the TSR diagnostics reveals the unique characteristics of MILD combustion: the explosive dynamics is more widely distributed in the broad preheat zone. Furthermore, by comparing the reactive $\Omega_{R}$ and extended $\Omega_{R+D}$ TSR, the regions of explosive dynamics are further distinguished between reaction-dominant and the reactive-diffusive dominant regions. These features will be useful in understanding the turbulent MILD flame structure as discuss in the following.

\section{DNS: Computational Setup}

For the analysis of turbulent MILD flames, we used the DNS data of a lean methaneair mixture combustion diluted with exhaust gas recirculation (EGR) [16-18, 20], in which details of the configuration and parametric conditions can be found. A brief summary is provided as follows.

The computational domain is cubic of size $L_{x} \times L_{y} \times L_{z}=10 \times 10 \times 10 \mathrm{~mm}^{3}$, discretised using a uniform $384 \times 384 \times 384$ mesh points. In the $x$-direction, the inflow and outflow boundary conditions were imposed utilizing Navier-Stokes characteristic boundary conditions [69], while all other directions were set as periodic.

The DNS is split into two stages, namely mixing DNS and combustion DNS, to mimic the EGR process involved in MILD combustion. For the mixing DNS, a fully developed homogeneous isotropic turbulence field is obtained first by performing non-reacting turbulence DNS in a periodic domain. Subsequently, a homogeneous scalar field is obtained by specifying a scalar energy spectrum as in [70], which is taken as the reaction progress variable field based on the fuel mass fraction, varying from 0 to 1 . The species mass fractions obtained from a one-dimensional freely propagating laminar flame with desired thermochemical conditions, pre-computed by 1D calculations, are then superimposed onto the homogeneous progress variable field. Finally, these scalar and velocity fields are allowed to evolve for about one large eddy turnover time in a periodic domain without any chemical reaction, in order to represent the exhaust gas mixing, for a duration much shorter than the reference autoignition delay time for the chosen mixture conditions. The recirculating exhaust gas (burnt gas of the above superimposed 1D flame) includes all species being present in the burnt gas of the 1D flame, such as reactants, products, radicals and intermediate species, which is subsequently fed from the inflow boundary of the MILD combustion domain. The energy equation is also solved during this mixing process, which creates a maximum temperature fluctuation of about $2 \%$ of the mean value. The generated scalar and velocity fields are used as the initial and inflow fields for the combustion DNS.

Table 2 summarizes the physical parameter values for the DNS case referred to as B1[16], where $S_{L}$ is the laminar flame speed, $\tau_{D}$ is the flow-through time, $\delta_{F}=\left(\lambda / \rho c_{p}\right) / S_{L}$ is the 
Table 2: List of the inflow turbulence parameters.

\begin{tabular}{cccccccc}
\hline$\delta_{t h}(\mathrm{~m})$ & $\tau_{D}(\mathrm{~ms})$ & $u^{\prime} / S_{L}$ & $l_{T} / \delta_{t h}$ & $l_{T} / \delta_{F}$ & $R e_{t}$ & $D a$ & $K a$ \\
\hline $0.94 \times 10^{-3}$ & 0.308 & 9.88 & 1.15 & 6.8 & 96.1 & 0.69 & 11.9 \\
\hline
\end{tabular}

Zeldovich flame thickness, where $\lambda$ and $c_{p}$ are respectively the mixture thermal conductivity and specific heat capacity at constant pressure, $\delta_{t h}=\left(T_{p}-T_{r}\right) / \max |\nabla T|_{L}$ is the thermal flame thickness, $D a=l_{T} \delta_{F} / u^{\prime} S_{L}$ is the Damköhler number, $K a=\left(u^{\prime} / S_{L}\right)^{3 / 2}\left(l_{T} / \delta_{F}\right)^{-1 / 2}$ is the Karlovitz number, and $R e_{t}=u^{\prime} l_{T} / \nu_{0}$ is the turbulent Reynolds number, where $\nu_{0}$ is the unburned gas kinematic viscosity.

Considering that the turbulent flow is statistically stationary and the lateral boundary conditions are periodic, the analysis was performed at an arbitrary mid-z plane at $t=0.9$ $\mathrm{ms} \approx 3 \tau_{D}$, i.e, when the initial transients have left the domain. Note that a few different planes were also analyzed and statistically consistent results were found.

\section{Explosive Dynamics in Turbulent MILD Combustion}

Figure 5 shows the spatial distributions of $\mathrm{CH}_{4}, \mathrm{O}_{2}, \mathrm{CO}_{2}, \mathrm{HCO}$ and $\mathrm{OH}$ mass fractions, along with temperature for DNS data. Typical characteristics of MILD combustion is seen in that the temperature increases only by $130 \mathrm{~K}$, in contrast to typical flames where difference is $1500 \mathrm{~K}$ or higher. According to the profiles of the reactants $\mathrm{CH}_{4}, \mathrm{O}_{2}$ and $\mathrm{CO}_{2}$, the spatial inhomogeneity in the mixture composition is evident as a result of the strong turbulent field. As will be shown later, the system's explosive dynamics does not relate to high concentration species like $\mathrm{CO}_{2}$. The mass fractions of $\mathrm{HCO}$ and $\mathrm{OH}$ are common flame/reaction zone markers used for turbulent flames. Unlike in normal flames where HCO and OH layers serve as a clear indicator of major reaction zones, the two species show significantly different pictures in MILD conditions in that their structures are more diffuse and widespread in the upstream region where turbulence is stronger. This clearly demonstrates that the conventional flame markers based on intermediate species isocontours do not work effectively in MILD combustion.

The corresponding TSR behavior on this turbulent MILD flame field is next investigated. As noted earlier, the extended TSR, $\Omega_{R+T}$ is computed based on Eq. 8 including the convective terms, as it is difficult to identify the mean convective velocity in such a nonuniform flow field. Nevertheless, the convective transport due to small scale turbulent eddies is properly accounted for.

Figure 6 shows the instantaneous distribution of $\Omega_{R+T}$ and $\Omega_{R}$ for the same solution field shown in Fig. 5. In contrast to the $0 \mathrm{D}$ and $1 \mathrm{D}$ cases, in the turbulent MILD combustion case $\Omega_{R}$ is negative everywhere, indicating that the net effect of all CSP modes when accounting for solely chemical kinetics is dissipative. When transport is taken into account, however, there are regions where $\Omega_{R+T}$ becomes positive, suggesting that the dominant dynamics there is explosive. In this region, one or more explosive modes are enhanced by transport, leading to positive $\Omega_{R+T}$.

To further investigate the nature of the explosive dynamics, $\Omega_{R+T}$ and $\Lambda_{e, f}$ are compared in Fig. 7 , along with the heat release rate. Similar to that in the $0 \mathrm{D}$ and $1 \mathrm{D}$ cases, $\Lambda_{e, f}$ exists 
in a broader region in the DNS case and does not represent the intense heat release region. On the other hand, Fig. 7 shows that the HRR is well correlated with the $\Omega_{R+T}$, since positive $\Omega_{R+T}$ is in general characterized by high HRR values. This finding suggests that the use of $\Lambda_{e, f}$ alone as an indicator of the system's dominant dynamics may be misleading in describing turbulent flame characteristics. Furthermore, the main distinct point in the $\Omega_{R+T}$ behavior in DNS is that it becomes dissipative in much of the preheat zone where diffusion was found to drive explosive dynamics in the 1D laminar flames. It is evident that the additional convective transport associated with small scale turbulent eddies significantly affects the flame dynamics.

To ascertain the role of convection in the explosive dynamics, Fig. 8 shows the region of $\Omega_{R+T}>0$ compared against the region of $\Omega_{R+D}>0$. Unlike in the $1 \mathrm{D}$ flame, the diffusive transport is no longer a major contributor to the explosive dynamics, so that $\Omega_{R+D}$ hardly becomes positive. Rather, it is the convective transport that drives the explosive modes, although such regions are more confined near the high heat release region.

The dominant role of turbulent convective transport in the flame dynamics is further elaborated by the TSR-API metric in Fig. 9, by comparing $\mathrm{H}_{\text {Chem }}^{R+T}, \mathrm{H}_{\text {Conv }}^{R+T}$, and $\mathrm{H}_{\text {Diff }}^{R+T}$, the contributions of reaction, convection, and diffusion to the dynamics (both explosive and dissipative) of $\Omega_{R+T}$, respectively. The $\Omega_{R+T}=0$ isoline is overlaid. Again, the contribution of diffusion is insignificant everywhere. $\mathrm{H}_{\text {Chem }}^{R+T}$ is dominant in most regions, varying approximately from 50 to $100 \%$, which is reasonable since the temperature is sufficiently high to justify the enhanced activation of all relevant chemistry. However, within the explosive $\Omega_{R+T}$ regions (denoted by the isolines), its contribution is reduced and supplemented by the convective contribution $\mathrm{H}_{C o n v}^{R+T}$, approximately up to $40 \%$. These results clearly suggest that, although chemistry is the dominant component of the system's dynamics, the turbulent convective transport makes the difference and becomes important in driving the explosive nature of $\Omega_{R+T}$. This is actually due to convective processes which render the amplitude of the explosive mode large enough so that the explosive time scale dominates the characteristic time scale. As will be shown next, it is mainly the convection of $\mathrm{CH}_{4}$ which enhances the amplitude of the explosive mode in these regions.

Figure 6 also illustrates the topology characteristics of the MILD combustion. At the parametric condition under study, the heat release zone as well as the positive $\Omega_{R+T}$ regions appear as blobs rather than contiguous layers, suggesting that the distributed combustion regime is realized. To further visualize the evidence, Fig. 10 shows $\Omega_{R+T}$ profiles against $c_{T}$ as a scatter plot as well as its average conditioned over $c_{T}$. The reference 1D laminar flame case is also overlaid. The fluctuations in $\Omega_{R+T}$ is large in the preheat zone, where some data points reach the level of the 1D flame condition. However, as a statistical average there is no clear indication that $\Omega_{R+T}$ exhibits a peak in the $c_{T}$ axis. This confirms that the MILD flame structure departs significantly from the flamelet regime, such that the progressive variable no longer serves as a proper marker to follow the strong reaction zones.

Another sign of turbulence affecting the chemical dynamics in the MILD flame is shown in Fig. 11 where the number of exhausted (fast) modes, $M$, as a function of $c_{T}$ for the DNS data is compared with that for the 1D flame. While a typical 1D MILD flame shows a number of exhausted modes that increases from 4 to 11 during the reaction progress, for the turbulent MILD combustion case there are no exhausted modes throughout the bulk of the flame $\left(c_{T}<0.8\right)$. That is, the intense turbulent eddies stir up the reaction dynamics 
throughout the entire flame zones, such that there are no sufficiently fast reactive modes anymore. In fact, the absence of exhausted modes indicates that the transport time scales become as fast as the fastest chemical time scales. Consequently, the mixture composition results entirely off the slow invariant manifold, with fast processes trying to relax the system onto it, explaining why $\Omega_{R}$ has large negative values.

A unique value of the TSR analysis in MILD combustion is also highlighted in Fig. 12 where the examination of the topology using $\Omega_{R+T}>0$ as marker of the reaction zones identifies unambiguously the regions characterized by auto-ignition and deflagration fronts. Within these regions, through the additional evaluation of the TSR-API related to chemistry and transport, i.e., $\mathrm{H}_{\text {Chem }}^{R+T}$ and $\mathrm{H}_{\text {Tran }}^{R+T}$, respectively, further distinguishes the regions where heat release is dominated by auto-ignition or deflagration phenomena depending on the

relative magnitudes of $\mathrm{H}_{\text {Chem }}^{R+T}$ and $\mathrm{H}_{\text {Tran }}^{R+T}$. In three different instantaneous solution fields shown in Fig. 12, it is found that autoignition prevails as expected for the MILD combustion, but it is remarkable that a significant level of deflagration fronts still persist. The TSR analysis presented in the present study effectively identifies the complex reactive structures within the MILD combustion which often exhibits mixed-mode combustion.

\section{Chemical Pathways for Explosive Dynamics}

To further investigate the underlying chemical pathways responsible for the competition between explosive and dissipative dynamics, the contributions of the CSP modes to the $\Omega_{R+T}$ were calculated throughout the entire domain, with an emphasis on the parts of the unburnt mixture and the reaction zone, as established by the positive values of the $\Omega_{R+T}$. Table 3 summarizes the CSP modes with the largest contributions to $\Omega_{R+T}$ at five points (only contributions more than $10 \%$ are shown), along with the largest values of the CSP API diagnostic tool. These points are representative of the targeted regions in the computational domain, i.e. the parts of the unburnt mixture and the reaction zone, and cover a wide range of the temperature based progress variable $\mathrm{c}_{T}$ and physical spaces. In particular, $\mathrm{P}_{1}$ is a point where $\Omega_{R+T}<0$ and $c_{T}=0.2$. As it will be shown next for $c_{T}>0.1, \Omega_{R+T}$ may be either positive or negative. Therefore, points $\mathrm{P}_{2}$ and $\mathrm{P}_{4}$ have been selected in order to represent this phenomenon, i.e., for the same $\mathrm{c}_{T}$ value (i.e., 0.3), $\Omega_{R+T}<0$ at $\mathrm{P}_{2}$, while it is positive at $\mathrm{P}_{4}$. Finally, at $\mathrm{P}_{3}$ and $\mathrm{P}_{5}, \mathrm{c}_{T}$ is increased to 0.4 and 0.5 , respectively, but $\Omega_{R+T}$ is negative and positive, respectively. As it will be shown in the next section, $\Omega_{R+T}$ has positive values mainly for $c_{T}<0.55$.

Table 3 demonstrates that $\Omega_{R+T}$ is mainly controlled by two CSP modes, i.e., mode 1 and 10, while mode 6 plays a secondary role. The action of modes 1 and 10 appears to be a competition, since the former is strongly dissipative, while the latter is strongly explosive. Although the explosive mode might have the largest contribution (even marginally), as is the case at $\mathrm{P}_{2}$, the net effect is negative due to the combined effect of dissipative modes (e.g., modes 1 and 6). That is, the dominant dynamics is dissipative in nature. The profile of $\Omega_{R+T}$ reveals that this competition leans mostly in favor of the dissipative dynamics, as is the case at points $\mathrm{P}_{1}-\mathrm{P}_{3}$, while points $\mathrm{P}_{4}$ and $\mathrm{P}_{5}$ are representative of where the explosive dynamics prevails.

Having identified the most significant CSP modes describing the system's slow dynamics, the next step is to identify the physical processes related to these CSP modes and determine 
Table 3: The CSP modes with the largest contributions to $\Omega_{R+T}$, at five selected points, along with the largest values of API of the dominant modes. Red/black font color represents explosive/dissipative modes, respectively.

\begin{tabular}{|c|c|c|c|c|c|c|c|c|c|}
\hline \multicolumn{2}{|l|}{$\mathrm{P}_{1}$} & \multicolumn{2}{|l|}{$\mathrm{P}_{2}$} & \multicolumn{2}{|c|}{$\mathrm{P}_{3}$} & \multicolumn{2}{|l|}{$\mathrm{P}_{4}$} & \multicolumn{2}{|l|}{$\mathrm{P}_{5}$} \\
\hline \multicolumn{10}{|c|}{ Mode contribution to $\Omega_{R+T}$} \\
\hline $\begin{aligned} \mathrm{c}_{T} & =0 . \\
\omega_{\tilde{\tau}_{R+T}} & =-\end{aligned}$ & 66.2 & $\begin{array}{r}\mathrm{c}_{T}=0 \\
\omega_{\tilde{\boldsymbol{\tau}}_{R+T}}=\end{array}$ & 46.1 & $\begin{array}{r}\mathrm{c}_{T}= \\
\omega_{\tilde{\boldsymbol{\tau}}_{R+T}}=\end{array}$ & $\begin{array}{l}4 \\
665.73\end{array}$ & $\begin{array}{r}\mathrm{c}_{T}=0 \\
\omega_{\tilde{\boldsymbol{\tau}}_{R+T}}=\end{array}$ & 78.4 & $\begin{array}{r}\mathrm{c}_{T}=0 \\
\omega_{\tilde{\boldsymbol{\tau}}_{R+T}}=\end{array}$ & 93.9 \\
\hline Mode 1 & $55 \%$ & Mode 10 & $42 \%$ & Mode 1 & $79 \%$ & Mode 10 & $63 \%$ & Mode 10 & $69 \%$ \\
\hline Mode 10 & $39 \%$ & $\begin{array}{l}\text { Mode } 1 \\
\text { Mode } 6\end{array}$ & $\begin{array}{l}41 \% \\
13 \%\end{array}$ & Mode 10 & $18 \%$ & Mode 1 & $19 \%$ & Mode 1 & $27 \%$ \\
\hline \multicolumn{10}{|c|}{ API of the dominant modes } \\
\hline \multicolumn{10}{|c|}{ Mode 10} \\
\hline $\mathrm{R} 1$ & $26 \%$ & $\mathrm{R} 1$ & $20 \%$ & $\mathrm{R} 1$ & $33 \%$ & Conv- $\mathrm{CH}_{4}$ & $17 \%$ & $\mathrm{R} 1$ & $25 \%$ \\
\hline R16 & $-9 \%$ & Conv- $\mathrm{O}_{2}$ & $12 \%$ & R16 & $-11 \%$ & $\mathrm{R} 1$ & $15 \%$ & Conv- $\mathrm{CH}_{4}$ & $12 \%$ \\
\hline Conv- $\mathrm{CH}_{4}$ & $8 \%$ & R16 & $-9 \%$ & R17 & $-10 \%$ & Conv- $\mathrm{O}_{2}$ & $-7 \%$ & R16 & $-7 \%$ \\
\hline R17 & $-7 \%$ & $\mathrm{Conv}^{-\mathrm{CH}_{4}}$ & $-8 \%$ & & & $\mathrm{R} 16$ & $-6 \%$ & Conv- $\mathrm{O}_{2}$ & $-7 \%$ \\
\hline \multicolumn{10}{|c|}{ Mode 1} \\
\hline R23 & $44 \%$ & $\mathrm{R} 23$ & $45 \%$ & $\mathrm{R} 23$ & $44 \%$ & R23 & $44 \%$ & R23 & $43 \%$ \\
\hline $\mathrm{R} 25$ & $-40 \%$ & R25 & $-40 \%$ & R25 & $-40 \%$ & $\mathrm{R} 25$ & $-40 \%$ & $\mathrm{R} 25$ & $-39 \%$ \\
\hline
\end{tabular}

their role to the system's explosive/dissipative characters. Starting with mode 1, the API tool in Fig. 13 and Table 3 show that its amplitude $\left(h^{1}\right)$ is mainly generated by the chain propagation reaction $\mathrm{R} 23\left(\mathrm{CH}_{2} \mathrm{O}+\mathrm{OH} \rightarrow \mathrm{HCO}+\mathrm{H}_{2} \mathrm{O}\right)$ and the dissociation reaction $\mathrm{R} 25$ $(\mathrm{HCO}+\mathrm{M} \rightarrow \mathrm{CO}+\mathrm{H}+\mathrm{M})$; the former favoring and the latter opposing the development of $h^{1}$. Note that these reactions are related to the production and consumption of HCO, a very important radical in the fuel oxidation of methane, and their heat release has been commonly used as flame marker in premixed combustion [71, 72]. In addition, both Fig. 13 and Table 3 confirm that reaction R25 is the dominant contributor to the timescale of the 1st mode $\tau_{1}$, strongly favoring its dissipative character. Note that the TPI and API of R23 and R25 do not exhibit noticeable differences in the computational domain. In addition, although not shown here, the largest API and TPI of the 6th mode are similar to those reported for the 1st mode, implying that these reactions expand to longer timescales.

On the other hand, Fig. 14 and Table 3 show that the amplitude of the fast explosive mode $10\left(h^{e, f}\right)$ is mainly generated by the chain branching reaction $\mathrm{R} 1$, while secondary contributions are provided by the convection of $\mathrm{CH}_{4}$, reaction $\mathrm{R} 16$ and the convection of $\mathrm{O}_{2}$. These findings are qualitatively in agreement with the $1 \mathrm{D}$ laminar flame case, where the amplitudes were associated with convection and reaction. In that case the convection of $\mathrm{CH}_{4}$ was highlighted along with the convection of $\mathrm{H}_{2}, \mathrm{CH}_{3}$ and reaction $\mathrm{R} 1$. By comparing the API profiles of reaction $\mathrm{R} 1$ and the convection of $\mathrm{CH}_{4}$ with the region where $\Omega_{R+T}>0$, it can be seen that $\Omega_{R+T}$ becomes positive in the regions where both convection of $\mathrm{CH}_{4}$ and reaction $\mathrm{R} 1$ are significant, enhancing the explosive mode's impact (i.e., $h^{e, f}$ ) to the system's slow evolution. This confirms the previous finding in Fig. 9 about the significant role of convection to the determination of the positive $\Omega_{R+T}$ values. This may be attributed to the system's enhanced dilution which acts against the ignitability of the mixture. Thus, the explosive character of the system is favored when a sufficient quantity of the fuel, e.g., $\mathrm{CH}_{4}$, is transported locally. In the absence of large gradients, diffusion becomes unimportant while 
convection becomes the only viable transport mechanism that can render such a change. Note also that in some regions where $\Omega_{R+T}>0$, the importance of methane convection becomes higher compared to that of reaction R1, in terms of the API values (see for instance P4 and $\mathrm{P} 5$ ). This further highlights the critical role of the methane convection to the establishment of explosive dynamics.

Additionally, Fig. 15 shows that the timescale of the 10th mode $\left(\tau_{f, e}\right)$ is mainly generated by reactions $\mathrm{R} 1$ and $\mathrm{R} 2$, both hydrogen-related, the former favoring and the latter opposing the explosive character of $\tau_{e, f}$. This is in agreement with the laminar flame case. Note that the region where $\Lambda_{f, e}$ exists is in complete agreement with the topology where R1 has large contribution to the mode's timescale, i.e., the fast explosive timescale, $\tau_{f, e}$.

\section{Conclusions}

Computational simulations representing a MILD combustion condition of methane/air mixtures were analyzed by using the CSP/TSR algorithmic tools to characterize their chemical and transport dynamics. As an overarching conclusion, for a general reactive/transport system such as laminar and turbulent flames, the TSR metric, as part of the CSP theory, is a more systematic and consistent way to identify the explosive dynamics of the system rather than examining the positive eigenvalues (chemically explosive modes) alone. 1D MILD flame structure further substantiate this fact in that the high temperature upstream region is made explosive through the interaction of reaction and diffusion, such that the chemical explosive metric alone does not properly capture its correct dynamical nature.

The analysis of turbulent DNS data revealed that the system's dynamics is mostly dissipative in nature, as the chemically explosive modes are largely suppressed by the dissipative action of transport. On the other hand, the convective transport associated with turbulent eddies play a key role in bringing the explosive nature into the system. As such $\Omega_{R+T}$ serves as the appropriate quantity to identify the regions of significant dynamical activities. In the turbulent MILD conditions under study, the flame structure appears nearly in the distributed combustion regime, such that the conventional statistics conditioned over the progress variable becomes inappropriate, but TSR serves as an automated and systematic way to depict the topology of such complex flames.

Further analysis of the CSP modes with the largest contributions to $\Omega_{R+T}$ revealed that the explosive mode is mostly favored by hydrogen-related reactions and the convection of $\mathrm{CH}_{4}$, while in the dissipative modes carbon-related reactions are found to be the most significant processes. As a result, the competition between these two different sets of reactions and the convection of $\mathrm{CH}_{4}$ are identified to be not only the key processes but also the main reason for the competitive action of the most important CSP modes, which results in a limited region of explosive dynamics in the computational domain, despite the extended existence of explosive modes.

The TSR/CSP tools employed in the present study successfully identified the explosive and dissipative states of the system and the dominant processes that contribute to the CSP modes and their related timescales, thus providing a systematic and automated means to

enhance our physical understanding of MILD combustion. Future work will explore a way to utilize this conceptual framework in turbulent combustion submodels. 


\section{Acknowledgments}

This work was sponsored by competitive research funding from King Abdullah University of Science and Technology (KAUST). Y.M. acknowledges the support of JSPS Grants-inAid for Scientific Research Grant Number JP19K14903. The authors would like to thank Professor Mauro Valorani at the Sapienza University of Rome for his continuing collaboration on this topic and valuable comments.

\section{References}

[1] A. Cavaliere, M. de Joannon, Mild combustion, Prog. Energy Combust. Sci. 30 (4) (2004) 329-366.

[2] M. Lackner, F. Winter, A. K. Agarwal, Handbook of combustion, Methods 1 (2013) 4.

[3] J. Wünning, J. Wünning, Flameless oxidation to reduce thermal no-formation, Progress in energy and combustion science 23 (1) (1997) 81-94.

[4] P. Sabia, M. de Joannon, A. Picarelli, R. Ragucci, Methane auto-ignition delay times and oxidation regimes in mild combustion at atmospheric pressure, Combust. Flame 160 (1) (2013) 47-55.

[5] M. De Joannon, A. Cavaliere, T. Faravelli, E. Ranzi, P. Sabia, A. Tregrossi, Analysis of process parameters for steady operations in methane MILD combustion technology, Proc. Combust. Inst. 30 (2) (2005) 2605-2612.

[6] P. Sabia, M. L. Lavadera, G. Sorrentino, P. Giudicianni, R. Ragucci, M. de Joannon, $\mathrm{H}_{2} \mathrm{O}$ and $\mathrm{CO}_{2}$ dilution in MILD combustion of simple hydrocarbons, Flow Turbul. Combust. 96 (2) (2016) 433-448.

[7] P. Sabia, G. Sorrentino, A. Chinnici, A. Cavaliere, R. Ragucci, Dynamic behaviors in methane MILD and oxy-fuel combustion. chemical effect of $\mathrm{CO}_{2}$, Energ. Fuel. 29 (3) (2015) 1978-1986.

[8] A. Mardani, S. Tabejamaat, S. Hassanpour, Numerical study of $\mathrm{CO}$ and $\mathrm{CO}_{2}$ formation in $\mathrm{CH}_{4} / \mathrm{H}_{2}$ blended flame under mild condition, Combust. Flame 160 (9) (2013) 16361649.

[9] T. Plessing, N. Peters, J. G. Wünning, Laseroptical investigation of highly preheated combustion with strong exhaust gas recirculation, in: Symposium (International) on combustion, Vol. 27-2, Elsevier, 1998, pp. 3197-3204.

[10] P. R. Medwell, P. A. Kalt, B. B. Dally, Simultaneous imaging of OH, formaldehyde, and temperature of turbulent nonpremixed jet flames in a heated and diluted coflow, Combust. Flame 148 (1-2) (2007) 48-61.

[11] M. De Joannon, A. Saponaro, A. Cavaliere, Zero-dimensional analysis of diluted oxidation of methane in rich conditions, Proceedings of the Combustion Institute 28 (2) (2000) 1639-1646. 
[12] N. Krishnamurthy, P. Paul, W. Blasiak, Studies on low-intensity oxy-fuel burner, Proceedings of the Combustion Institute 32 (2) (2009) 3139-3146.

[13] C. Duwig, B. Li, Z. Li, M. Aldén, High resolution imaging of flameless and distributed turbulent combustion, Combustion and Flame 159 (1) (2012) 306-316.

[14] I. Özdemir, N. Peters, Characteristics of the reaction zone in a combustor operating at mild combustion, Experiments in fluids 30 (6) (2001) 683-695.

[15] Y. Minamoto, T. Dunstan, N. Swaminathan, R. Cant, DNS of EGR-type turbulent flame in MILD condition, Proc. Combust. Inst. 34 (2) (2013) 3231-3238.

[16] Y. Minamoto, N. Swaminathan, S. R. Cant, T. Leung, Morphological and statistical features of reaction zones in MILD and premixed combustion, Combust. Flame 161 (11) (2014) 2801-2814.

[17] Y. Minamoto, N. Swaminathan, R. S. Cant, T. Leung, Reaction zones and their structure in MILD combustion, Combust. Sci. Techn. 186 (8) (2014) 1075-1096.

[18] Y. Minamoto, N. Swaminathan, Scalar gradient behaviour in MILD combustion, Combust. Flame 161 (4) (2014) 1063-1075.

[19] Y. Minamoto, N. Swaminathan, Subgrid scale modelling for MILD combustion, Proc. Combust. Inst. 35 (3) (2015) 3529-3536.

[20] N. A. K. Doan, N. Swaminathan, Y. Minamoto, Dns of mild combustion with mixture fraction variations, Combustion and Flame 189 (2018) 173-189.

[21] M. U. Göktolga, J. A. van Oijen, L. P. H. de Goey, 3d dns of mild combustion: a detailed analysis of heat loss effects, preferential diffusion, and flame formation mechanisms, Fuel 159 (2015) 784-795.

[22] S. Lam, D. Goussis, Understanding complex chemical kinetics with computational singular perturbation, Proc. Combust. Inst. 22 (1) (1989) 931-941.

[23] M. Valorani, S. Paolucci, E. Martelli, T. Grenga, P. P. Ciottoli, Dynamical system analysis of ignition phenomena using the tangential stretching rate concept, Combust. Flame 162 (8) (2015) 2963-2990.

[24] D. M. Manias, A.-E. Tingas, H. G. Im, Y. Minamoto, Dynamics analysis of a turbulent methane flame in mild combustion conditions, in: AIAA Scitech 2019 Forum, 2019, p. 1731.

[25] S. Lam, D. Goussis, The CSP method for simplifying kinetics, Int. J. Chem. Kinet. 26 (4) (1994) 461-486.

[26] D. M. Manias, E. A. Tingas, C. E. Frouzakis, K. Boulouchos, D. A. Goussis, The mechanism by which $\mathrm{CH}_{2} \mathrm{O}$ and $\mathrm{H}_{2} \mathrm{O}_{2}$ additives affect the autoignition of $\mathrm{CH}_{4}$ /air mixtures, Combust. Flame 164 (2016) 111-125. 
[27] M. Jaasim, E.-A. Tingas, F. E. Hernández Pérez, H. G. Im, Computational singular perturbation analysis of super-knock in SI engines, Fuel 225 (2018) 184-191.

[28] W. Song, E.-A. Tingas, H. G. Im, A computational analysis of methanol autoignition enhancement by dimethyl ether addition in a counterflow mixing layer, Combust. Flame 195 (2018) 84-98.

[29] E.-A. Tingas, D. C. Kyritsis, D. A. Goussis, $\mathrm{H}_{2}$ /air autoignition dynamics around the third explosion limit, J. Energ. Eng. 145 (1) (2018) 04018074.

[30] D. J. Diamantis, E. Mastorakos, D. A. Goussis, $\mathrm{H}_{2} /$ air autoignition: The nature and interaction of the developing explosive modes, Combust. Theor. Model. 19 (3) (2015) 382-433.

[31] M. Valorani, D. A. Goussis, Explicit time-scale splitting algorithm for stiff problems: auto-ignition of gaseous mixtures behind a steady shock, J. Comput. Phys. 169 (1) (2001) 44-79.

[32] D. A. Goussis, Quasi steady state and partial equilibrium approximations: their relation and their validity, Combust. Theor. Model. 16 (5) (2012) 869-926.

[33] M. Valorani, P. P. Ciottoli, R. M. Galassi, S. Paolucci, T. Grenga, E. Martelli, Enhancements of the g-scheme framework, Flow, Turbulence and Combustion (2018) 1-11.

[34] D. A. Goussis, G. Skevis, Nitrogen chemistry controlling steps in methane-air premixed flames, in: K. J. Bathe (Ed.), Comput. Fluid Solid Mech., Elsevier, Amsterdam, 2005, pp. 650-653.

[35] D. A. Goussis, H. N. Najm, Model reduction and physical understanding of slowly oscillating processes: the circadian cycle, Multiscale Model. Simul. 5 (4) (2006) 12971332.

[36] D. M. Manias, D. J. Diamantis, D. A. Goussis, Algorithmic identification of the reactions related to the initial development of the time scale that characterizes $\mathrm{CH}_{4} /$ air autoignition, Journal of Energy Engineering 141 (2) (2014) C4014015.

[37] Y. Li, A. Alfazazi, B. Mohan, E. A. Tingas, J. Badra, H. G. Im, S. M. Sarathy, Development of a reduced four-component (toluene/n-heptane/iso-octane/ethanol) gasoline surrogate model, Fuel 247 (2019) 164-178.

[38] E. A. Tingas, D. M. Manias, S. M. Sarathy, D. A. Goussis, CH $\mathrm{CH}_{4} /$ air homogeneous autoignition: A comparison of two chemical kinetics mechanisms, Fuel 223 (2018) 7485.

[39] M. Hadjinicolaou, D. A. Goussis, Asymptotic solution of stiff PDEs with the CSP method: The reaction diffusion equation, SIAM J. Sci. Comput. 20 (3) (1998) 781-810.

[40] M. Valorani, F. Creta, D. A. Goussis, -local and global manifolds in stiff reactiondiffusion systems, in: Computational Fluid and Solid Mechanics 2003, Elsevier, 2003, pp. $1548-1551$. 
[41] M. Valorani, H. N. Najm, D. A. Goussis, CSP analysis of a transient flame-vortex interaction: time scales and manifolds, Combust. Flame 134 (1) (2003) 35-53.

[42] D. A. Goussis, M. Valorani, F. Creta, H. N. Najm, Reactive and reactive-diffusive time scales in stiff reaction-diffusion systems, Prog. Comput. Fluid Dy. 5 (6) (2005) 316-326.

[43] H. N. Najm, M. Valorani, D. A. Goussis, J. Prager, Analysis of methane-air edge flame structure, Combust. Theor. Model. 14 (2) (2010) 257-294.

[44] D. Goussis, S. Lam, A study of homogeneous methanol oxidation kinetics using csp, Proc. Combust. Inst. 24 (1) (1992) 113-120.

[45] E. A. Tingas, D. C. Kyritsis, D. A. Goussis, Autoignition dynamics of DME/air and EtOH/air homogeneous mixtures, Combust. Flame 162 (9) (2015) 3263-3276.

[46] M. Valorani, F. Creta, F. Donato, H. N. Najm, D. A. Goussis, Skeletal mechanism generation and analysis for n-heptane with CSP, Proc. Combust. Inst. 31 (1) (2007) 483-490.

[47] A. Massias, D. Diamantis, E. Mastorakos, D. Goussis, An algorithm for the construction of global reduced mechanisms with CSP data, Combust. Flame 117 (4) (1999) 685-708.

[48] E.-A. Tingas, D. J. Diamantis, D. A. Goussis, Issues arising in the construction of QSSA mechanisms: the case of reduced n-heptane/air models for premixed flames, Combust. Theor. Model. 22 (6) (2018) 1049-1083.

[49] S. M. Sarathy, E.-A. Tingas, E. F. Nasir, A. Detogni, Z. Wang, A. Farooq, H. Im, Three-stage heat release in n-heptane auto-ignition, Proc. Combust. Inst. 37 (1) (2019) $485-492$.

[50] E.-A. Tingas, Z. Wang, S. M. Sarathy, H. G. Im, D. A. Goussis, Chemical kinetic insights into the ignition dynamics of n-hexane, Combust. Flame 188 (2018) 28-40.

[51] G. Skevis, D. Goussis, E. Mastorakos, Understanding methane flame kinetics from reduced mechanisms, Int. J. Alternat. Propul. 1 (2-3) (2007) 216-227.

[52] J. Prager, H. N. Najm, M. Valorani, D. Goussis, Structure of n-heptane/air triple flames in partially-premixed mixing layers, Combust. Flame 158 (11) (2011) 2128-2144.

[53] M. Kooshkbaghi, C. E. Frouzakis, K. Boulouchos, I. V. Karlin, n-heptane/air combustion in perfectly stirred reactors: Dynamics, bifurcations and dominant reactions at critical conditions, Combust. Flame 162 (9) (2015) 3166-3179.

[54] I. A. Dodoulas, S. Navarro-Martinez, Analysis of extinction in a non-premixed turbulent flame using large eddy simulation and the chemical explosion mode analysis, Combust. Theor. Model. 19 (1) (2015) 107-129.

[55] P. Pal, M. Valorani, P. G. Arias, H. G. Im, M. S. Wooldridge, P. P. Ciottoli, R. M. Galassi, Computational characterization of ignition regimes in a syngas/air mixture with temperature fluctuations, Proc. Combust. Inst. 36 (3) (2017) 3705-3716. 
[56] D. Manias, A.-E. Tingas, F. E. Hernández Pérez, H. G. Im, R. Malpica Galassi, P. P. Ciottoli, M. Valorani, Analysis of hydrogen/air turbulent premixed flames at different Karlovitz numbers using computational singular perturbation, in: 2018 AIAA Aerospace Sciences Meeting, 2018, p. 0364.

[57] D. M. Manias, E.-A. Tingas, F. E. H. Pérez, R. M. Galassi, P. P. Ciottoli, M. Valorani, H. G. Im, Investigation of the turbulent flame structure and topology at different karlovitz numbers using the tangential stretching rate index, Combust. Flame 200 (2019) $155-167$.

[58] E. A. Tingas, D. C. Kyritsis, D. A. Goussis, Ignition delay control of DME/air and $\mathrm{EtOH} /$ air homogeneous autoignition with the use of various additives, Fuel 169 (2016) $15-24$.

[59] E. A. Tingas, D. C. Kyritsis, D. A. Goussis, Algorithmic determination of the mechanism through which $\mathrm{H}_{2} \mathrm{O}$-dilution affects autoignition dynamics and $\mathrm{NO}$ formation in $\mathrm{CH}_{4}$ /air mixtures, Fuel 183 (2016) 90-98.

[60] E. A. Tingas, H. G. Im, D. C. Kyritsis, D. A. Goussis, The use of $\mathrm{CO}_{2}$ as an additive for ignition delay and pollutant control in $\mathrm{CH}_{4}$ /air autoignition, Fuel 211 (2018) 898-905.

[61] D. J. Diamantis, D. C. Kyritsis, D. A. Goussis, The reactions favoring or opposing the development of explosive modes: auto-ignition of a homogeneous methane/air mixture, Proc. Combust. Inst. 35 (2015) 267-274.

[62] E.-A. Tingas, D. C. Kyritsis, D. A. Goussis, Comparative investigation of homogeneous autoignition of DME/air and EtOH/air mixtures at low initial temperatures, Combust. Theor. Model. 21 (1) (2017) 93-119.

[63] M. Valorani, P. P. Ciottoli, R. M. Galassi, Tangential stretching rate (TSR) analysis of non premixed reactive flows, Proc. Combust. Inst. 36 (1) (2017) 1357-1367.

[64] CSPTk - a software toolkit for the CSP and TSR analysis of kinetic models and the simplification and reduction of chemical kinetics mechanisms. The software can be obtained upon request to M.Valorani (mauro.valorani@uniroma1.it), Tech. rep., Sapienza University of Rome, Italy (2015).

[65] C. Safta, H. N. Najm, O. Knio, TChem-a software toolkit for the analysis of complex kinetic models, Sandia Report, SAND2011-3282.

[66] M. D. Smooke, Reduced kinetic mechanisms and asymptotic approximations for methane-air flames, Lecture Notes in Physics 384.

[67] GRI-Mech; release 3.0, http://www.me.berkeley.edu/gri_mech (2000).

[68] A. Chemkin-Pro, 17.2, ANSYS Reaction Design: San Diego.

[69] T. J. Poinsot, S. Lelef, Boundary conditions for direct simulations of compressible viscous flows, J. Comput. Phys. 101 (1) (1992) 104-129. 
[70] V. Eswaran, S. Pope, Direct numerical simulations of the turbulent mixing of a passive scalar, The Physics of fluids 31 (3) (1988) 506-520.

[71] Z. M. Nikolaou, N. Swaminathan, Heat release rate markers for premixed combustion, Combustion and Flame 161 (12) (2014) 3073-3084.

[72] I. A. Mulla, A. Dowlut, T. Hussain, Z. M. Nikolaou, S. R. Chakravarthy, N. Swaminathan, R. Balachandran, Heat release rate estimation in laminar premixed flames using laser-induced fluorescence of ch2o and h-atom, Combustion and Flame 165 (2016) 373383.

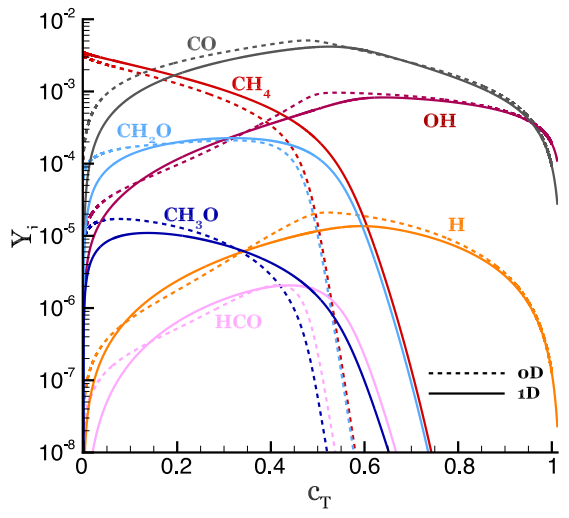

(a)

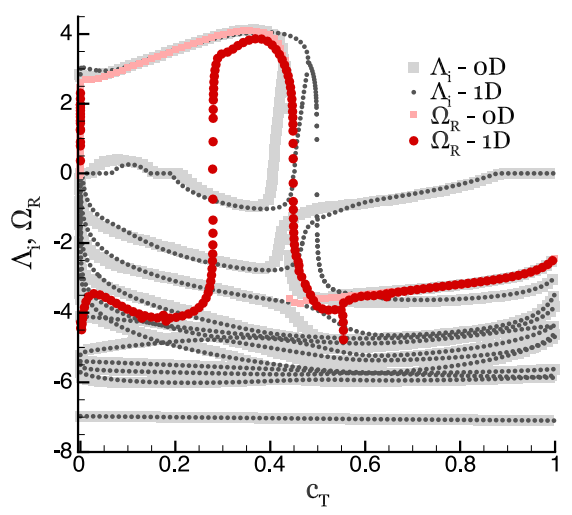

(b)

Figure 1: The evolution of selected species mass fractions (a) and the eigenvalues $\Lambda_{i}$ along with the reactive TSR, $\Omega_{R}$ (b), for the 0D autoignition and the $1 \mathrm{D}$ laminar cases, against $c_{T}$.

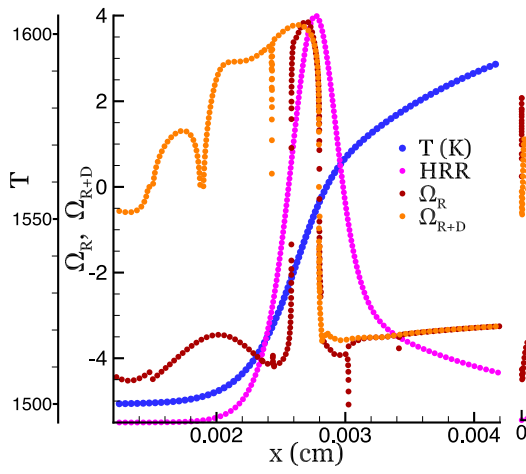

(a)

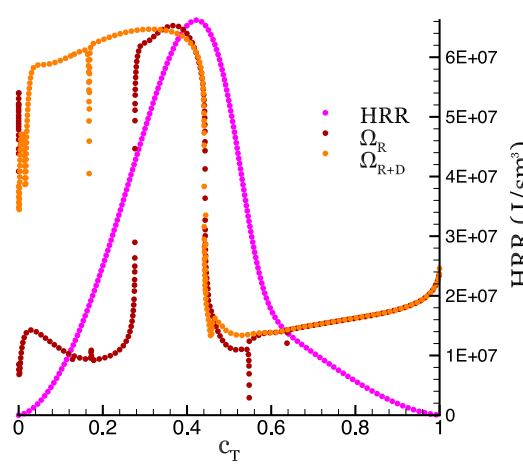

(b)

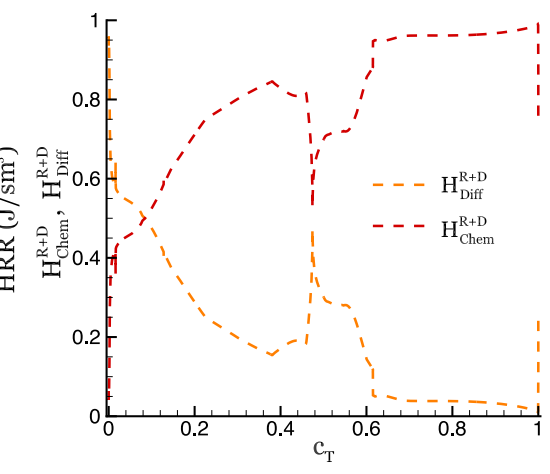

(c)

Figure 2: The evolution in the physical (a) and progress variable, $\mathrm{c}_{T}$, (b) spaces of $\Omega_{R}$ and heat release rate, HRR $[\mathrm{J} /(\sec \times \mathrm{m})]$, along with $\Omega_{R+D}$ for the $1 \mathrm{D}$ case. In (a) blue represents the temperature profile. (b) The variation of $H_{C h e m}^{R+D}$ and $H_{D i f f}^{R+D}$ for the $1 \mathrm{D}$ case, against $\mathrm{c}_{T}$. 


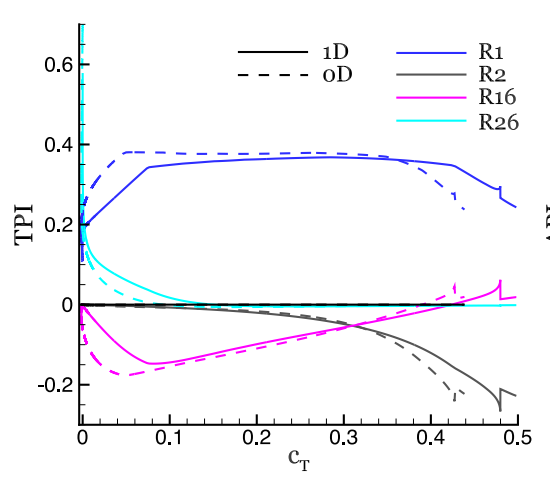

(a)

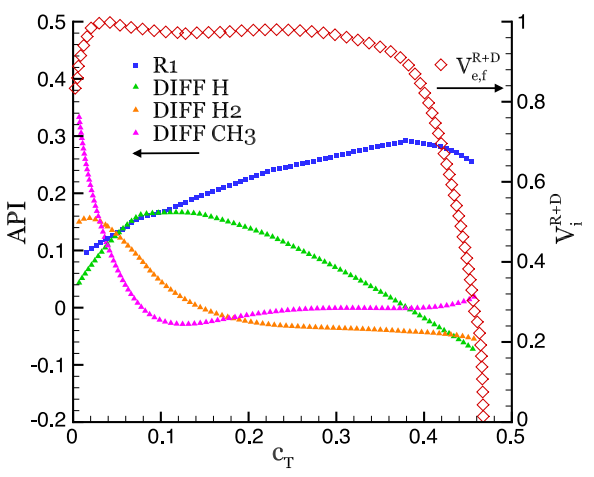

(b)

Figure 3: Evolution of the largest (a) TPI and (b) API values to the system's fast explosive mode, as a function of $c_{T}$. Figure (a) includes results for the 0D and 1D cases while figure (b) shows the results for only the $1 \mathrm{D}$ case and the contribution of the fast explosive mode to $\Omega_{R+D}$ (Eq. 16) has been overlaid.

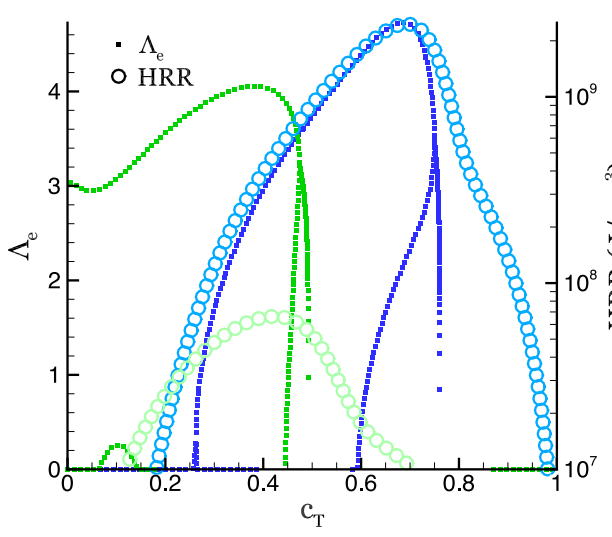

(a)

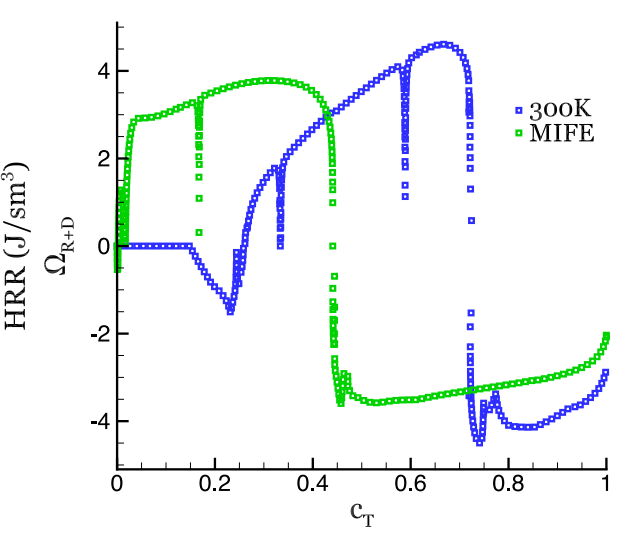

(b)

Figure 4: Spatial evolution of (a) $\Lambda_{e}$, overlaid with HRR, and (b) $\Omega_{R+D}$ as a function of $\mathrm{c}_{T}$ for the MILD (green) and normal flame (blue) at $\phi=0.8$ and $\mathrm{T}_{0}=300 \mathrm{~K}$. 


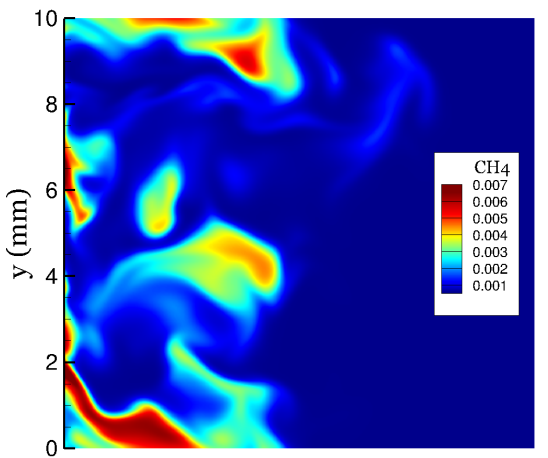

(a)

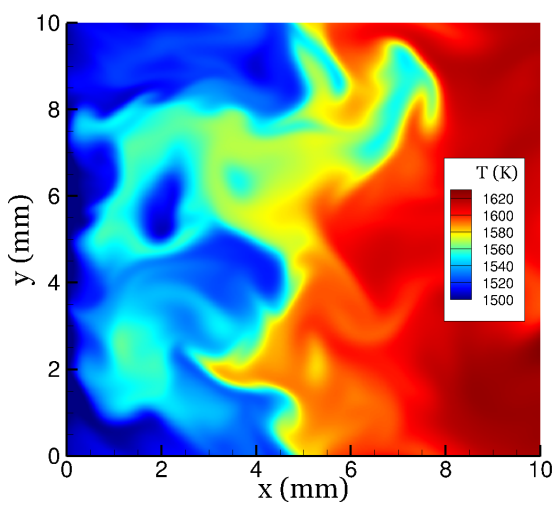

(d)

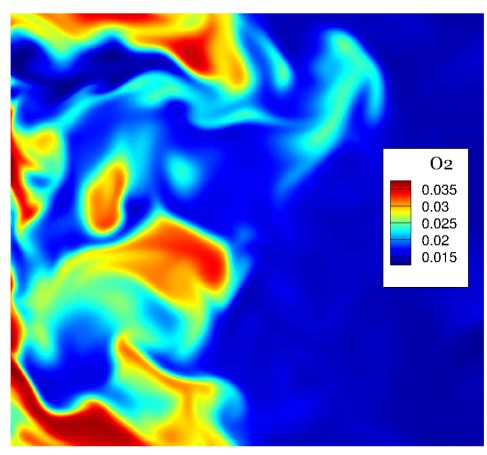

(b)

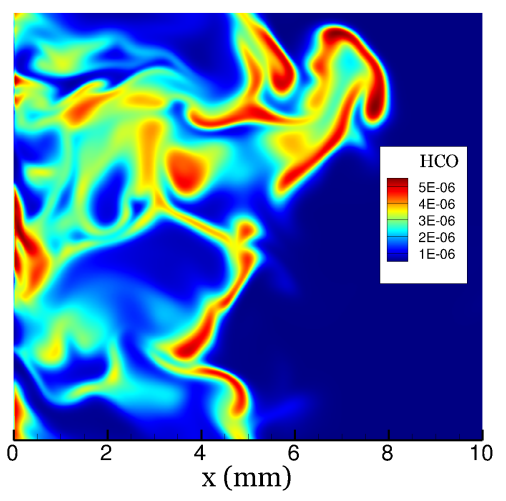

(e)

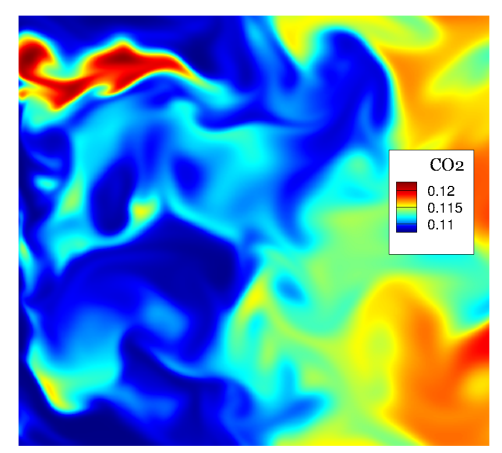

(c)

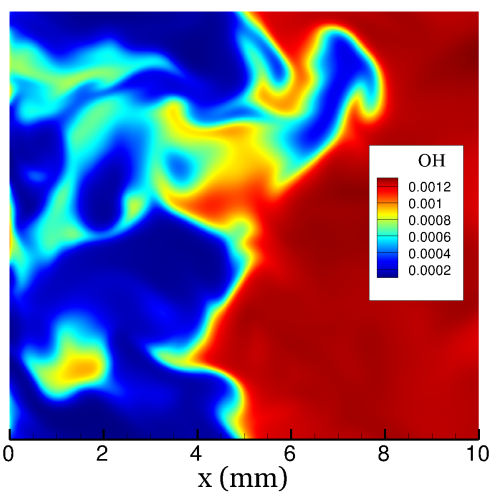

(f)

Figure 5: The mid-z plane spatial distribution of the mass fractions of the reactants $\mathrm{CH}_{2}$ (a), $\mathrm{O}_{2}$ (b) and $\mathrm{CO}_{2}$ (c), temperature (d) and the mass fractions of the usual HRR indicators $\mathrm{HCO}(\mathrm{e})$ and $\mathrm{OH}(\mathrm{f})$ for the DNS case at $t=0.9 \mathrm{~ms}$.

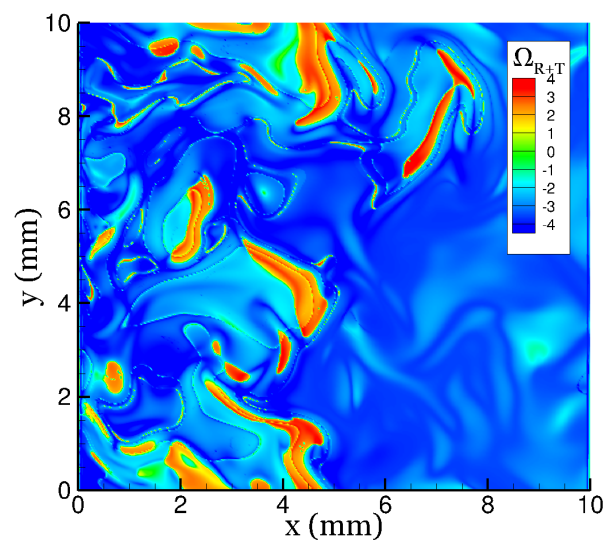

(a)

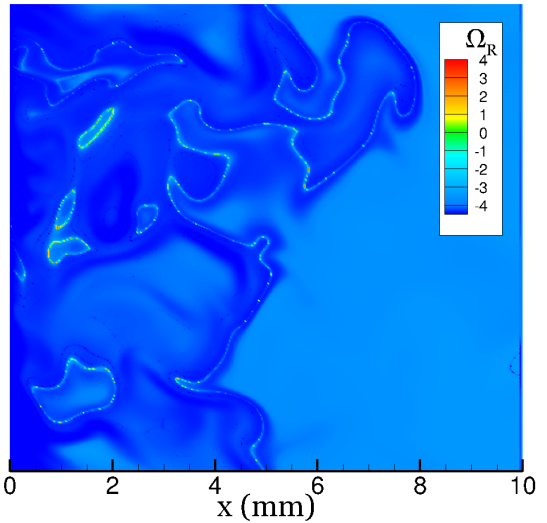

(b)

Figure 6: The mid-z plane spatial distributions of (a) $\Omega_{R+T}$ and (b) $\Omega_{R}$ for the DNS case at $t=0.9 \mathrm{~ms}$. 


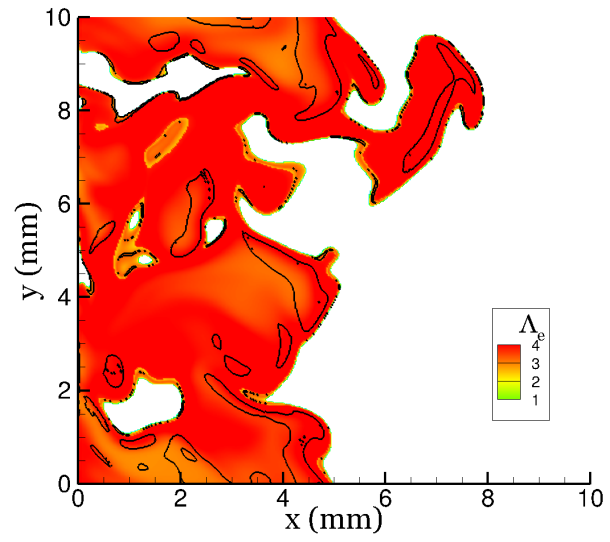

(a)

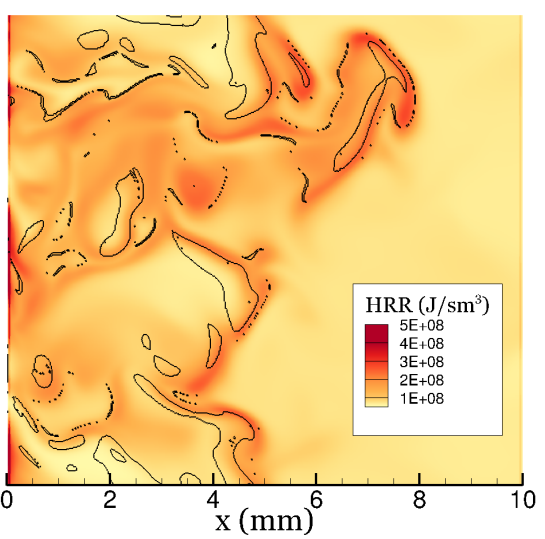

(b)

Figure 7: The mid-z plane spatial distributions of (a) $\Lambda_{e, f}$ and (b) the heat release rate $\left[\mathrm{J} /\left(\mathrm{sec} \times \mathrm{m}^{3}\right)\right]$, overlaid with the $\Omega_{R+T}=0$ black solid isoline for the DNS case at $t=0.9 \mathrm{~ms}$.

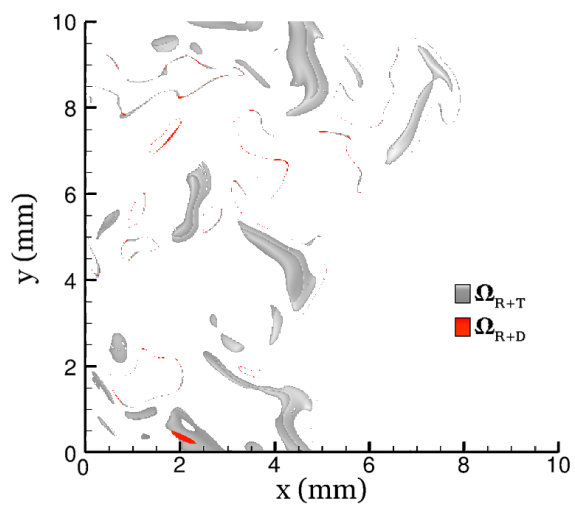

Figure 8: The mid-z plane spatial distribution of $\Omega_{R+T}>0$ overlaid with the $\Omega_{R+D}>0$ distribution for the DNS case at $t=0.9 \mathrm{~ms}$.

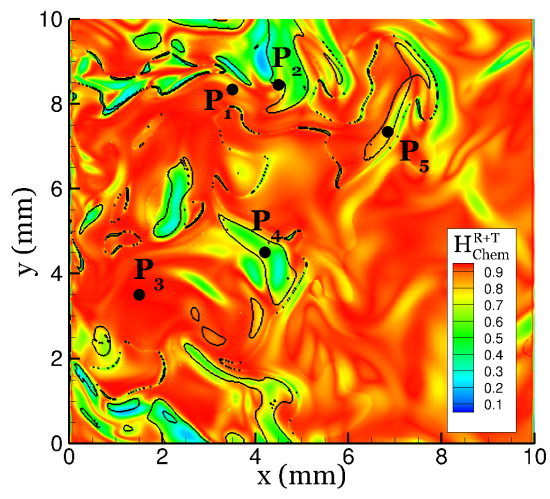

(a)

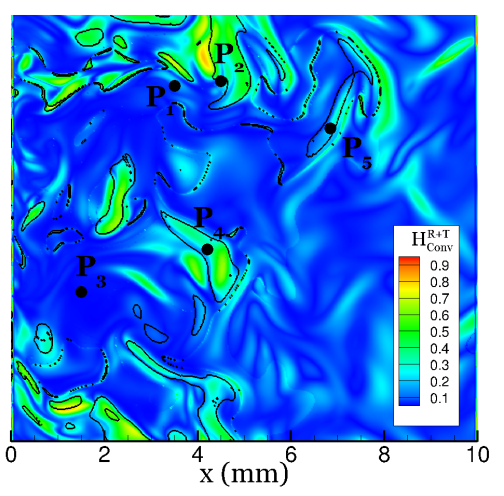

(b)

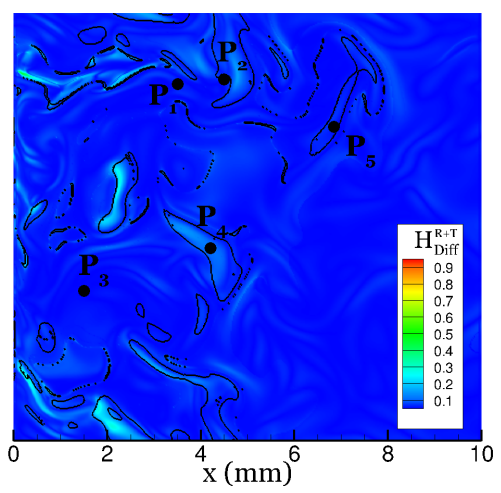

(c)

Figure 9: The mid-z plane spatial distributions of the $\mathrm{H}_{C h e m}^{R+T}$ (a), $\mathrm{H}_{\text {Conv }}^{R+T}$ (b) and $\mathrm{H}_{D i f f}^{R+T}$ (c) overlaid with the $\Omega_{R+T}=0$ isoline for the DNS case at $t=0.9 \mathrm{~ms}$. Points $\mathrm{P}_{1}-\mathrm{P}_{5}$ are analyzed for their mode make-ups in Table 3. 


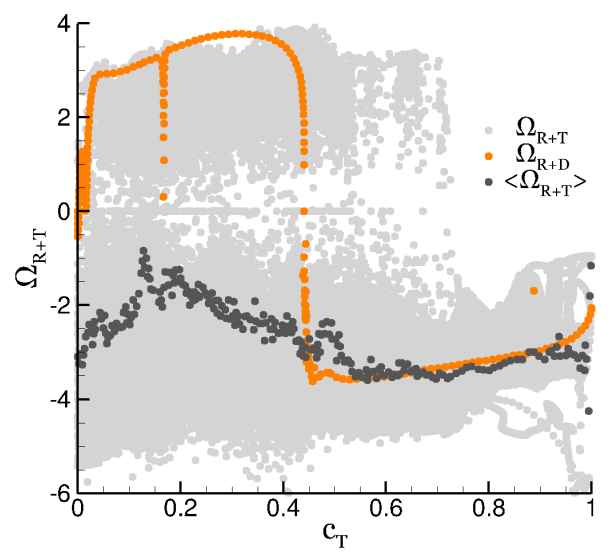

(a)

Figure 10: Local values of $\Omega_{R+T}$ and its value conditioned over $c_{T},\left\langle\Omega_{R+T}\right\rangle$, as a function of $c_{T}$ for the DNS case at $t=0.9 \mathrm{~ms}$, where the laminar values of $\Omega_{R+D}$ of the $1 \mathrm{D}$ case are overlaid.

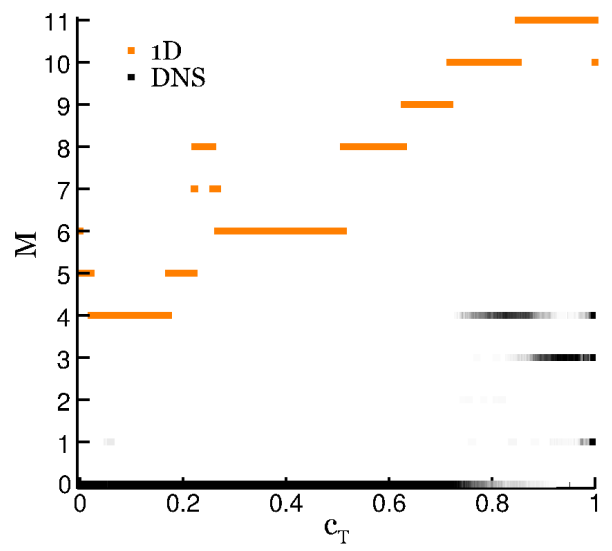

Figure 11: The number of exhausted modes, $\mathrm{M}$, for the 1D flame and for the DNS data as a function of $\mathrm{c}_{T}$.

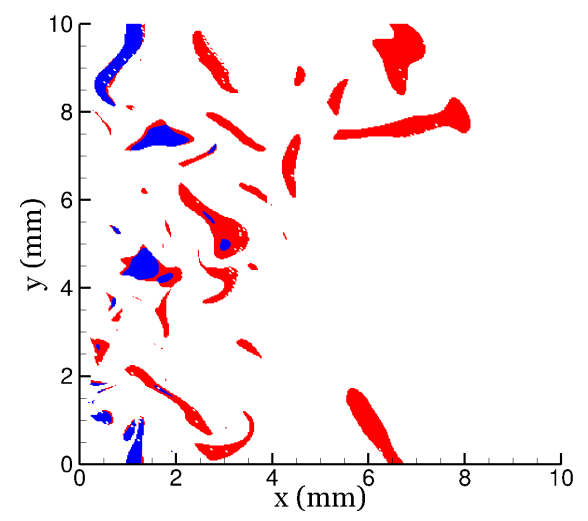

(a)

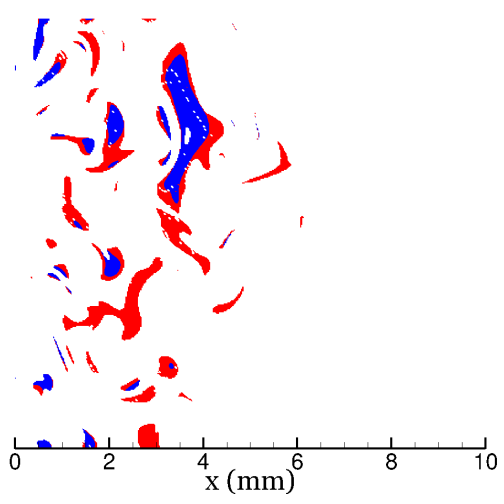

(b)

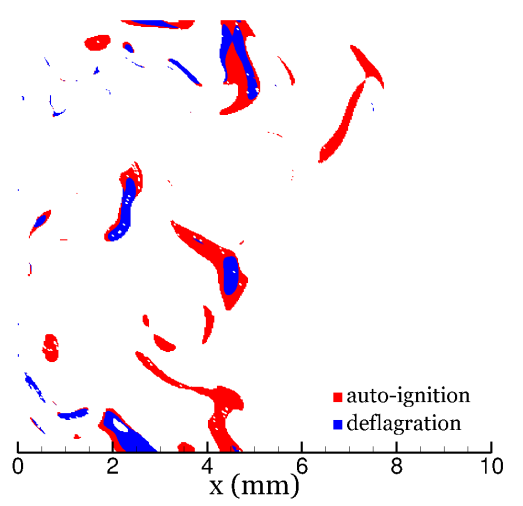

(c)

Figure 12: Auto-ignition vs deflagration regions within the region of positive $\Omega_{R+T}$ for the DNS data at three timesteps: (a) $t=0.7 \mathrm{~ms}$, (b) $t=0.8 \mathrm{~ms}$ and (c) $t=0.9 \mathrm{~ms}$. 


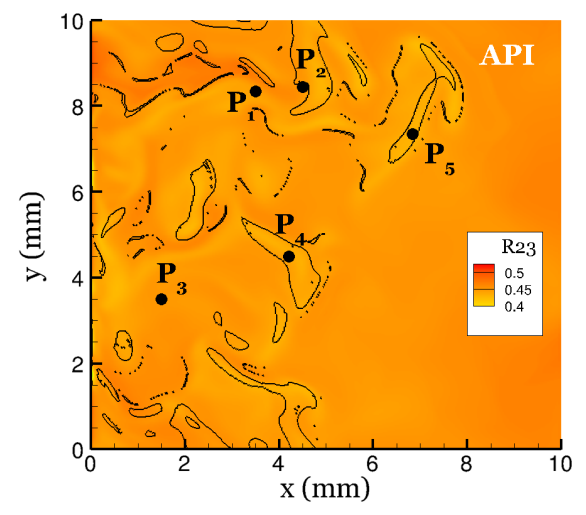

(a)

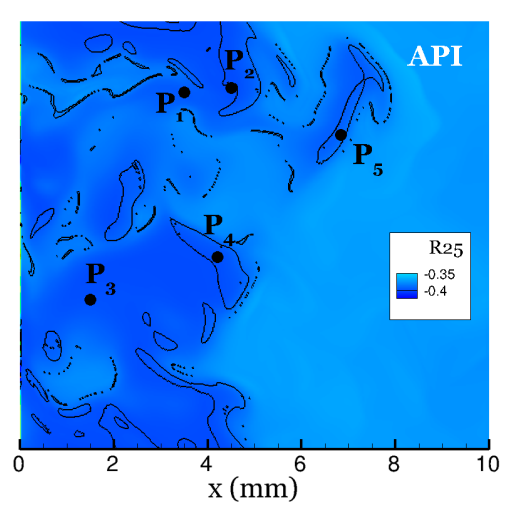

(b)

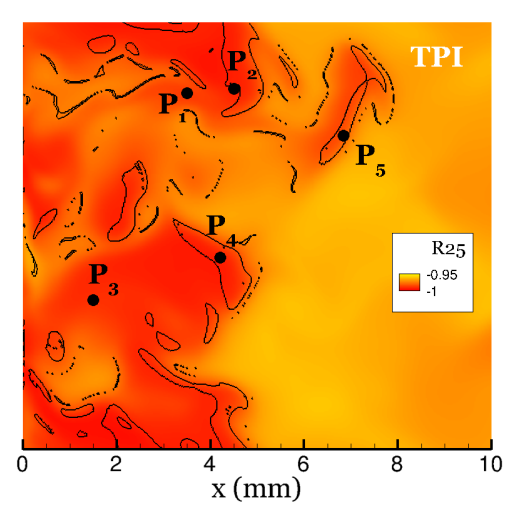

(c)

Figure 13: The mid-z plane spatial distribution of the processes with the largest API ((a) and (b)) and TPI (c) values for the dissipative mode 1 , overlaid with the $\Omega_{R+T}=0$ isoline, for the DNS case at $t=0.9$ ms. Points $\mathrm{P}_{1}-\mathrm{P}_{5}$ refer to Table 3.

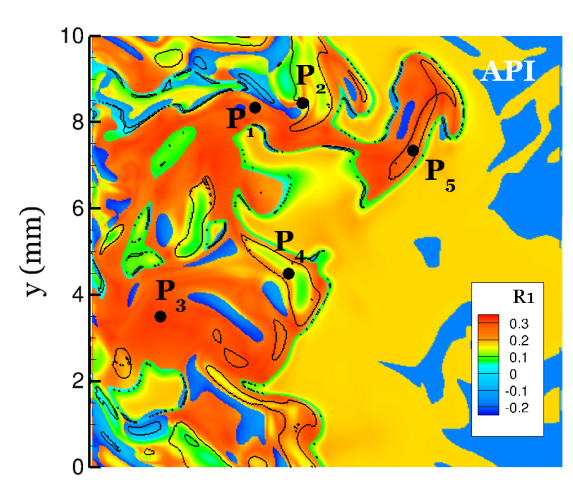

(a)

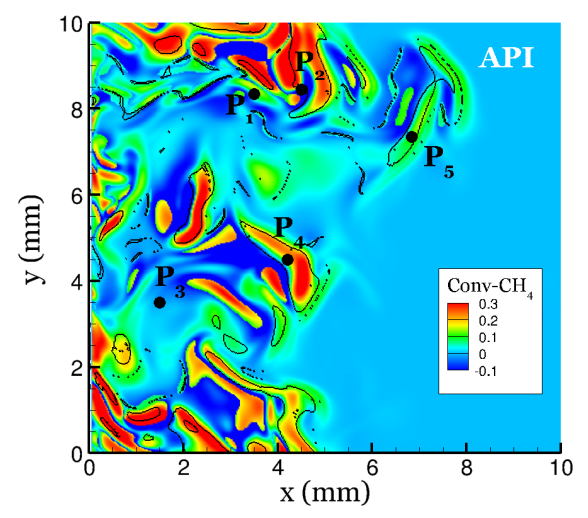

(c)

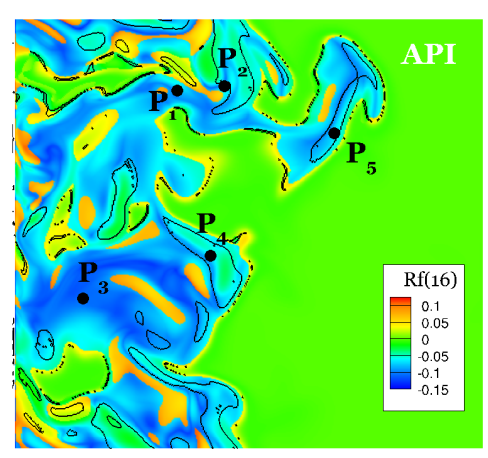

(b)

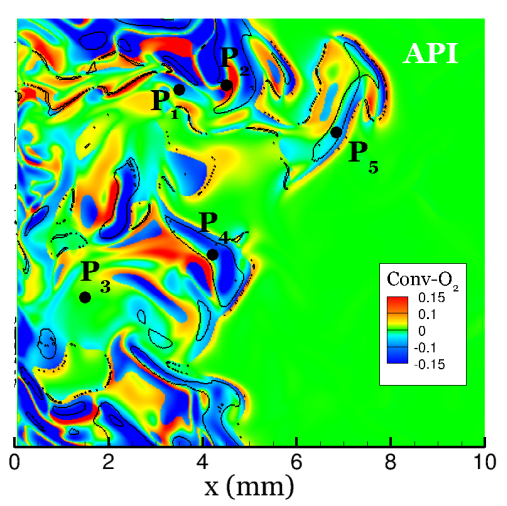

(d)

Figure 14: The mid-z plane spatial distribution of the processes with the largest API values for the fast explosive mode 10 overlaid with the $\Omega_{R+T}=0$ isoline, for the DNS case at $t=0.9 \mathrm{~ms}$; (a) reaction R1, (b) reaction R16, (c) convection of $\mathrm{CH}_{4}$, (d) convection of $\mathrm{O}_{2}$. Points $\mathrm{P}_{1}-\mathrm{P}_{5}$ refer to Table 3. 


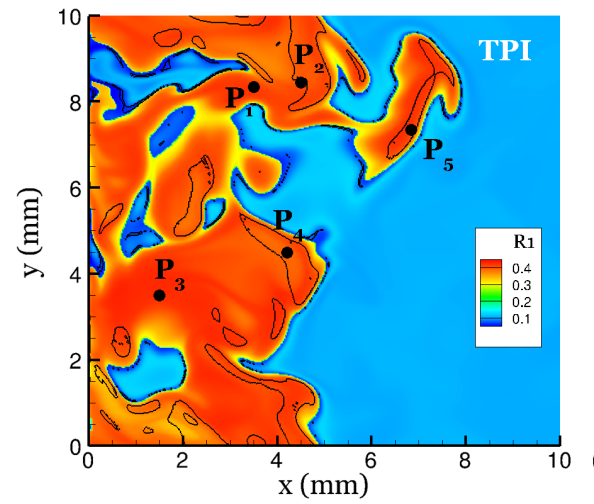

(a)

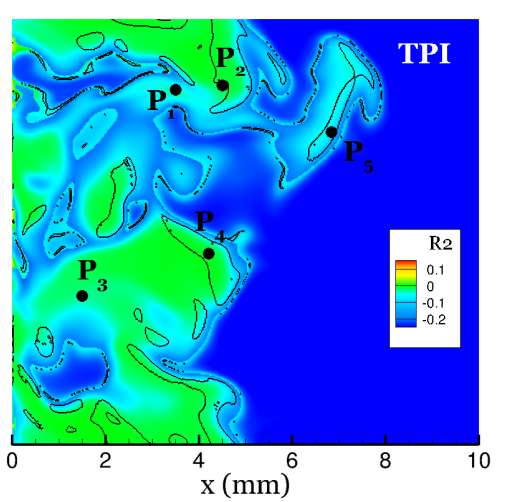

(b)

Figure 15: The mid-z plane spatial distribution of the processes with the largest TPI values for the fast explosive mode 10, overlaid with the $\Omega_{R+T}=0$ isoline, for the DNS case at $t=0.9 \mathrm{~ms}$; (a) reaction R1, (b) reaction R2. Points $\mathrm{P}_{1}-\mathrm{P}_{5}$ refer to Table 3 . 